\title{
Stability and chaotic behavior of a PID controlled inverted pendulum subjected to harmonic base excitations by using the normal form theory
}

\author{
Manuel F. Pérez-Polo ${ }^{a^{*}}$, Manuel Pérez Molina ${ }^{b}$, Javier Gil Chica ${ }^{c}$, José A. Berna \\ Galiano $^{\mathrm{d}}$
}

Departamento de Física, Ingeniería de Sistemas y Teoría de la Señal, Universidad de Alicante, Escuela Politécnica Superior, Campus de San Vicente, 03071, Alicante, Spain. Fax 34-9-659-09750, ${ }^{\mathrm{a}}$ Tel: 34-9-659-09673. E- mail: manolo@dfists.ua.es , ${ }^{\mathrm{b}} \mathrm{Tel}$ : 34-9659-02077. E-mail: ma_perez_m@hotmail.com, ${ }^{\mathrm{c}}$ Tel: 34-9-659-02077. E-mail: gil@dfists.ua.es, ${ }^{\mathrm{d}}$ Tel: 34-9-659-02077. E-mail: jberna@dfists.ua.es.

\begin{abstract}
In this paper we investigate the stability and the onset of chaotic oscillations around the pointing-up position for a simple inverted pendulum that is driven by a control torque and is harmonically excited in the vertical and horizontal directions. The driven control torque is defined as a proportional plus integral plus derivative (PID) control of the deviation angle with respect to the pointing-down equilibrium position. The parameters of the PID controller are tuned by using the Routh criterion to obtain a stable weak focus around the pointing-up position, whose stability is investigated by using the normal form theory. The normal form theory is also used to deduce a simplified mathematical model that can be resolved analytically and compared with the numerical simulation of the complete mathematical model. From the harmonic prescribed motions for the pendulum base, necessary conditions for chaotic motion are deduced by means of the Melnikov function. When the pendulum is close to the unstable pointing-up position, the PID parameters are changed and the chaotic motion is destroyed, which is achieved by employing very small control signals even in the presence of random noise. The results of the analytical calculations are verified by full numerical simulations.
\end{abstract}

Keywords: Simple pendulum, harmonic base excitation, PID control, normal from theory, Melnikov's method, chaotic motion.

* Author whose correspondence should be addressed 


\section{Introduction}

The problem of stability and dynamical behavior of an inverted pendulum subjected to harmonic vibrations in the suspension point is related to many fields of physics and engineering, such as vibrations of oscillatory chains, control theory, bifurcations, normal form theory and chaos, among others. The analysis of the dynamical behavior of a simple inverted pendulum has been studied in connection with stability problems, both from a theoretical and experimental viewpoint and with delay [1-6]. However, analytical solutions of the problem assuming oscillations in the suspension point are only considered under certain simplifications in the problem, as it appears in Ref [2].

On the other hand, the problem of swinging up and controlling a pendulum has been considered in the classical Refs [7-8]. Other more complex control strategies reveal the great interest of the inverted pendulum in the field of control, as it is the case of control strategies based on space-state methods [9-11], control stabilization around homoclinic orbits [12], energy methods [13], passivity control [14] and bounded control [15] among others. However, the use of a simple control law to obtain chaotic behavior has been less used. This is probably due to the difficulty in determining whether a pendulum with harmonic base excitations can exhibit chaotic dynamics [16-20].

The aim of this paper is to investigate the stability and dynamical behavior around the pointing-up position for a simple inverted pendulum that is driven by a control torque and is harmonically excited in the vertical and horizontal directions. It is known that a dissipative pendulum subjected to vertical and horizontal harmonic disturbances of high frequency can be driven to several equilibrium points apart from the stable pointing-down position (examples can be found in Refs [21-26]). Consequently, for a pendulum with high-frequency vertical oscillations in the suspension point, the unstable pointing-up position transforms into a stable one, whereas the pendulum can reach a stable tilt angle below $\pi / 2$ for high-frequency horizontal oscillations. In these cases, the required forces in the suspension point to maintain these equilibriums can be very large. What is more important, these equilibrium points depend on the initial conditions, and the presence of random noise 
could to destroy them. The previous reasoning will be used to justify the application of a simple control law based on a PID controller to swing-up and maintain the pendulum in the pointing-up position.

In the first part of this paper we assume that there are no harmonic disturbances, and the parameters values of the PID controller which lead to a weak focus in the pointing-up position are deduced from the Routh stability criterion. The stability of this weak focus is studied through the normal form theory, from which it is possible to deduce the system equations in normal form and compare them with the numerical simulations of the system. For this purpose, the method developed by Bruno [27-32] will be applied (other approaches can be found in refs. [33-36]). Once the system is reduced to its normal form, the stability properties associated to the parameter variations of the PID controller will be investigated.

The conditions which result in chaotic behavior for the pendulum without control torque when a harmonic motion is applied to the suspension point have also been analyzed through the Melnikov function [33-38]. The chaotic motion and the appearance of strange attractors are verified by means of the sensitive dependence, Lyapunov exponents, power spectral density and Poincaré sections [33-36], [38]. Taking into account the heteroclinic tangle in a strange attractor, there will always be trajectories in the phase plane that will be very close to the up right position. For such trajectories, the PID parameter values are properly changed so that the chaotic motion is destroyed and the pendulum is maintained around the pointing-up equilibrium position with small oscillations, even in the presence of random noise.

\section{Mathematical model and statement of the problem}

Fig. 1 shows the layout of the pendulum system as well as the notation used to deduce the Lagrangian of the system. The pendulum is modeled by a mass $\mathrm{m}$ hanging at the end of a rod of negligible mass and length 1 , which is fixed to a support $O$ [4-6], [716]. Let $\mathrm{O}^{\prime} X Y$ be an inertial frame and $F_{x 0}(t), F_{y 0}(t)$ the forces applied at the suspension point $\mathrm{O}^{\prime}$ in the $\mathrm{OX}$ and $\mathrm{OY}$ directions, which respectively produce the accelerations given by $\ddot{x}_{0}(t)$ and $\ddot{y}_{0}(t)$. 
Figure 1

The kinetic and potential energies of the system can be written as follows:

$$
\begin{gathered}
\left.\begin{array}{l}
x=x_{0}+l \sin \theta \\
y=y_{0}-l \cos \theta
\end{array}\right\} \Rightarrow T=\frac{1}{2} m\left[\left(\dot{x}_{0}^{2}+\dot{y}_{0}^{2}\right)+l^{2} \dot{\theta}^{2}+2 l \dot{\theta}\left(\dot{x}_{0} \cos \theta+\dot{y}_{0} \sin \theta\right)\right] \\
V=m g(l-l \cos \theta)+m g y_{0}
\end{gathered}
$$

From Eqs (1) and (2) the Lagrange function is obtained as:

$$
L=T-V=\frac{1}{2} m\left(\dot{x}_{0}^{2}+\dot{y}_{0}^{2}\right)+\frac{1}{2} m l \dot{\theta}^{2}+m l \dot{\theta}\left(\dot{x}_{0} \cos \theta+\dot{y}_{0} \sin \theta\right)-m g(l-l \cos \theta)-m g y_{0}
$$

Assuming a Rayleigh dissipation function $F_{r}=b \dot{\theta}^{2} / 2$ associated to the angular variable $\theta$, and taking into account the control torque $\mathrm{u}(\mathrm{t})$ as well as the forces $\mathrm{F}_{\mathrm{x} 0}$ and $\mathrm{F}_{\mathrm{y} 0}$ applied at the suspension point $\mathrm{O}^{\prime}$, the mathematical model of the system can be obtained from the Lagrange equations as:

$$
\begin{gathered}
m l^{2} \ddot{\theta}+m g l \sin \theta+b \dot{\theta}+m l\left(\ddot{x}_{0} \cos \theta+\ddot{y}_{0} \sin \theta\right)=u(t) \\
m \ddot{x}_{0}+m l \ddot{\theta} \cos \theta-m l \dot{\theta}^{2} \sin \theta=F_{x 0} \\
m \ddot{y}_{0}+m l \ddot{\theta} \sin \theta+m l \dot{\theta}^{2} \cos \theta+m g=F_{y 0}
\end{gathered}
$$

The term $m l\left(\ddot{x}_{0} \cos \theta+\ddot{y}_{0} \sin \theta\right)$ in Eq (4) is considered as an external disturbance, since it is assumed that the accelerations $\ddot{x}_{0}$ and $\ddot{y}_{0}$ of the mobile suspension point $\mathrm{O}^{\prime}$ are defined through the following harmonic motion:

$$
\left.\begin{array}{c}
x_{0}=A_{x} \sin \left(\omega_{x} t+\varphi\right) \\
y_{0}=A_{y} \sin \omega_{y} t
\end{array}\right\} \Rightarrow\left\{\begin{array}{c}
\ddot{x}_{0}=-A_{x} \omega_{x}^{2} \sin \left(\omega_{x} t+\varphi\right) \\
\ddot{y}_{0}=-A_{y} \omega_{y}^{2} \sin \omega_{y} t
\end{array}\right.
$$


where $A_{x}$ is the amplitude of the horizontal displacement of $\mathrm{O}^{\prime}, \omega_{\mathrm{x}}$ is the frequency of the horizontal motion component of $\mathrm{O}^{\prime}$ and $\varphi$ is an arbitrary phase shift, whereas $\mathrm{A}_{\mathrm{y}}$ and $\omega_{y}$ are the vertical displacement and the frequency of the vertical motion component for O' respectively. It should be noticed that Eq (4) can be numerically solved from a specified control torque $\mathrm{u}(\mathrm{t})$, and the forces $\mathrm{F}_{\mathrm{x} 0}$ and $\mathrm{F}_{\mathrm{y} 0}$ can be obtained from Eqs (5)(7). Next, it is assumed that the control torque is a PID controller, i.e. it is defined by [39]:

$$
u(t)=-K_{p}\left[[\theta(t)-\pi]+\frac{1}{\tau_{i}} \int_{0}^{t}[\theta(\tau)-\pi] d \tau+\tau_{d} \frac{d \theta(t)}{d t}\right]
$$

where $K_{p}, \tau_{i}$ and $\tau_{d}$ are the proportional action constant, the reset time and the derivative time respectively [39]. From Eqs (4) and (8), the mathematical model of the pendulum with harmonic base excitation and PID control can be written as follows:

$$
\begin{gathered}
\frac{d^{2} \theta(t)}{d t^{2}}+\omega_{0}^{2} \sin \theta(t)+\delta \frac{d \theta(t)}{d t}+\frac{1}{l}\left[\ddot{x}_{0} \cos \theta(t)+\ddot{y}_{0} \sin \theta(t)\right]=\alpha u(t) \\
\frac{d u(t)}{d t}=-\frac{K_{p}}{\tau_{i}}[\theta(t)-\pi]-K_{p} \frac{d \theta(t)}{d t}-K_{p} \tau_{d} \frac{d^{2} \theta(t)}{d t^{2}}
\end{gathered}
$$

where the following notation has been introduced:

$$
\omega_{0}^{2}=\frac{g}{l} ; \delta=\frac{b}{m l^{2}} ; \alpha=\frac{1}{m l^{2}}
$$

Now we are going to analyze the advantages and appropriateness of the simple control law defined by Eq (8). It is well known that the unstable pointing-up position of the pendulum can be transformed into a stable one when the suspension point is excited in the vertical direction at high frequencies [21-26]. Consequently, it is possible to make stable the pointing-up position by varying the amplitude $A_{y}$ and the frequency $\omega_{y}$ of the external disturbances [25-26]. To analyze this effect we shall consider a simplified case in which there are only vertical vibrations at $\mathrm{O}^{\prime}$, i.e $\mathrm{x}_{0}=0$. Since the control torque is now zero, from Eqs (7) and (9) it is deduced that: 


$$
\frac{d^{2} \theta(t)}{d t^{2}}+\omega_{0}^{2} \sin \theta(t)+\delta \frac{d \theta(t)}{d t}-\frac{A_{y} \omega_{y}^{2}}{l} \sin \omega_{y} t \sin \theta(t)=0
$$

By introducing the dimensionless time $\bar{t}=\omega_{0} t, \mathrm{Eq}(11)$ can be rewritten as:

$$
\frac{d^{2} \theta(\bar{t})}{d \bar{t}^{2}}+\sin \theta(\bar{t})+\frac{\delta}{\omega_{0}} \frac{d \theta(\bar{t})}{d \bar{t}}-\frac{A_{y} \omega_{y}^{2}}{l \omega_{0}^{2}} \sin \frac{\omega_{y}}{\omega_{0}} \bar{t} \sin \theta(\bar{t})=0
$$

By using the averaging method [25], [40] it is possible to separate the high frequency motion components from the low frequency motion components to obtain the governing equation for the slow motions, which can be expressed in terms of the variable $t$ as [25]:

$$
\frac{d^{2} \theta_{1}(t)}{d t^{2}}+\sin \theta_{1}(t)+\frac{\delta}{\omega_{0}} \frac{d \theta_{1}(t)}{d t}+\frac{1}{4} \frac{A_{y}^{2} \omega_{y}^{2}}{l^{2} \omega_{0}^{2}} \sin 2 \theta_{1}(t)=0
$$

where the angle $\theta_{1}$ accounts for the slow motions. In the pointing-up equilibrium position of the pendulum, the Jacobian of the system (13) and the corresponding eigenvalues are given by:

$$
J_{\theta_{1}=\pi}=\left|\begin{array}{cc}
0 & 1 \\
\left(1-\frac{1}{2} \frac{A_{y}^{2} \omega_{y}^{2}}{l^{2} \omega_{0}^{2}}\right) & -\frac{\delta}{\omega_{0}}
\end{array}\right| \Rightarrow \lambda_{1,2}=-\frac{\delta}{2 \omega_{0}} \pm \sqrt{\left(\frac{\delta}{2 \omega_{0}}\right)^{2}+\left(1-\frac{1}{2} \frac{A_{y}^{2} \omega_{y}^{2}}{l^{2} \omega_{0}^{2}}\right)}
$$

Consequently, the pointing-up equilibrium position will be stable if $A_{y} \omega_{y} / l \omega_{0}>\sqrt{2}$. Similarly, it can be shown that the pointing-down equilibrium position is stable for all values of $A_{y}$ and $\omega_{y}$. To study this issue, Eqs (9) have been simulated assuming that $A_{x}$ $=0, \mathrm{~A}_{\mathrm{y}}=0.5 \mathrm{~m}, 1=1 \mathrm{~m}, \omega_{0}=3.1305 \mathrm{~m} / \mathrm{s}^{2}$ and $\omega_{\mathrm{y}}=10$, so $A_{y} \omega_{L y} / l \omega_{0}=1.597>\sqrt{2}\left(\omega_{\mathrm{Ly}}\right.$ $=8.8544 \mathrm{rad} / \mathrm{s}$ is the limit frequency). The initial conditions are $\theta(0)=2.9 \mathrm{rad}, \mathrm{d} \theta(0) / \mathrm{dt}$ $=0$ and $\mathrm{u}(0)=0$ (i.e. they are close to the pointing-up position) and the simulation results are shown in Fig 2. Fig 2 a) shows the time evolution of $\theta(t), d \theta(t) / d t$ and $u(t) \equiv$ 0 . At $\mathrm{t} \approx 15 \mathrm{~s}$ the position $\theta=\pi$ is reached, whereas $\theta(\mathrm{t})$ and $\mathrm{d} \theta(\mathrm{t}) / \mathrm{dt}$ show an irregular behavior that suggests chaotic behavior for $\mathrm{t}<15 \mathrm{~s}$. To analyze this behavior, Fig $2 \mathrm{~b}$ ) depicts two simulations of Eqs (9) whose initial conditions differ in $10^{-7}$, which allows 
to appreciate a clear sensitive dependence as a strong indicator of chaotic behavior. Fig 2 c) shows the values of the forces $F_{0 x}$ and $F_{0 y}$ deduced from Eqs (5) and (6), which can be regarded as acceptable. On the other hand, we assume the presence of random noise in the system that is modeled as follows:

$$
\theta(t)=\theta(t)+f_{n a}[X-0.5] ; \mathrm{d} \theta(t) / d t=\mathrm{d} \theta(t) / d t+f_{n a}[X-0.5]
$$

where $X$ is a random variable that is uniformly distributed between 0 and 1 , and $\mathrm{f}_{\mathrm{na}}>0$ is an amplification factor to obtain a uniformly distributed noise amplitude between $\mathrm{f}_{\mathrm{na}} / 2$ and $\mathrm{f}_{\mathrm{na}} / 2$. For $\theta(0)=2.9 \mathrm{rad}, \mathrm{d} \theta(0) / \mathrm{dt}=0, \mathrm{u}(0)=0$ and $f_{n a}=0.2$, Fig $\left.2 \mathrm{~d}\right)$ shows that the desired set point $\theta_{\mathrm{e}}=\pi$ cannot be reached.

Figure 2

To analyze the effect of the PID control law given by Eq (8), Fig 3 shows the simulation results of Eqs (9) that have been obtained with the previous values but taking $\mathrm{K}_{\mathrm{p}}=30 \mathrm{~N} . \mathrm{m}, \tau_{\mathrm{i}}=1 \mathrm{~s}$ and $\tau_{\mathrm{d}}=10^{-3} \mathrm{~s}$, assuming the initial conditions $\theta(0)=0.01 \mathrm{rad}$, $\mathrm{d} \theta(0) / d t=0, u(0)=0$ and considering a noise factor $f_{n a}=0.4$. Figs 3 a) and $3 b$ ) show how the desired set point $\theta_{\mathrm{e}}=\pi$ is reached even with strong noise and very disadvantageous initial conditions due to the PID controller action. Fig 3 c) shows that the magnitude of the forces deduced from Eqs (5) and (6) are acceptable even in presence of the PID control torque.

Figure 3

It should be noticed that, although the PID parameters $\mathrm{K}_{\mathrm{p}}, \tau_{\mathrm{i}}$, and $\tau_{\mathrm{d}}$ have been chosen arbitrarily, the derivative time $\tau_{\mathrm{d}}$ must be small enough to avoid an excessive value for $\mathrm{u}(\mathrm{t})$ due to the high values of the derivatives caused by the random noise [3839]. The effect of the high-frequency horizontal excitation can be analyzed in a similar way. In this case, the averaged equation and the Jacobian of the pointing-down equilibrium position are given by [25]: 


$$
\begin{gathered}
\frac{d^{2} \theta_{1}(t)}{d t^{2}}+\sin \theta_{1}(t)+\frac{\delta}{\omega_{0}} \frac{d \theta_{1}(t)}{d t}-\frac{1}{4} \frac{A_{x}^{2} \omega_{x}^{2}}{l^{2} \omega_{0}^{2}} \sin 2 \theta_{1}(t)=0 \\
J_{\theta_{1}=\pi}=\left|\begin{array}{cc}
0 & 1 \\
-\left(1-\frac{1}{2} \frac{A_{x}^{2} \omega_{x}^{2}}{l^{2} \omega_{0}^{2}}\right) & -\frac{\delta}{\omega_{0}}
\end{array}\right| \Rightarrow \lambda_{1,2}=-\frac{\delta}{2 \omega_{0}} \pm \sqrt{\left(\frac{\delta}{2 \omega_{0}}\right)^{2}-\left(1-\frac{1}{2} \frac{A_{x}^{2} \omega_{x}^{2}}{l^{2} \omega_{0}^{2}}\right)}
\end{gathered}
$$

Therefore, the pointing-down equilibrium position will be unstable if $A_{x} \omega_{x} / l \omega_{0}>\sqrt{2}$. In this case, Eq (16) allows to deduce that two new equilibrium points appear in symmetric positions around $\theta=0$, i.e.:

$$
\theta_{e}= \pm \operatorname{arcos}\left(\frac{2 l^{2} \omega_{0}^{2}}{A_{x}^{2} \omega_{x}^{2}}\right)
$$

where the sign of $\theta_{\mathrm{e}}$ depends on the initial conditions [25-26]. Taking $\theta_{\mathrm{e}}=0.8 \mathrm{rad}, 1=1$ $\mathrm{m}, \omega_{0}=3.1305 \mathrm{rad} / \mathrm{s}$ and $\mathrm{A}_{\mathrm{x}}=0.035 \mathrm{~m}, \mathrm{Eq}(18)$ allows to deduce that $\omega_{\mathrm{x}}=151.54 \mathrm{rad} / \mathrm{s}$. As it can be observed in Fig 4 a), the simulation results for Eqs (9) indicate that the tilt angle of the pendulum is $\theta_{\mathrm{e}} \approx-0.8 \mathrm{rad}$, which is very close to the prescribed value. The small amplitude around the averaged value is a consequence of the large value for $\omega_{\mathrm{x}}$. In accordance with Eqs (5) and (6), Fig 4 b) shows the strong forces that must be applied to the suspension point $\mathrm{O}^{\prime}$ to maintain the reference angle for the pendulum.

\section{Figure 4}

The previous results allow to conclude that:

- It is possible to obtain chaotic behavior without PID control and with vertical excitations of moderate frequency.

- It is possible to drive the pendulum to the set point $\theta_{\mathrm{e}}=\pi$ without PID control and with vertical excitation, although the initial conditions must be close to the set point.

- Without PID control and with vertical excitation, a small random noise or a small change in the initial conditions may destroy the asymptomatically stable equilibrium point $\theta_{\mathrm{e}}=\pi$ (see Fig $2 \mathrm{a}$ )), and the pendulum behavior may become oscillatory around $\theta_{\mathrm{e}}=\pi$ as shown in Fig $2 \mathrm{~d}$ ). 
- Without PID control and with horizontal excitations of high frequency, the pendulum cannot be driven to the pointing-up position, even with very high frequencies or strong forces applied at the suspension point $\mathrm{O}^{\prime}$.

- Starting from arbitrary initial conditions, the pendulum can be driven to the pointing-up position with PID control and with vertical excitations of high frequency, even in the presence of random noise.

Once the need of a PID controller has been clarified, the following deviation variables are introduced:

$$
z_{1}^{\prime}(t)=\theta(t)-\pi ; z_{2}^{\prime}(t)=\dot{\theta}(t) ; z_{3}^{\prime}(t)=u(t)
$$

Removing the term $d^{2} \theta(t) / d t^{2}$ from the second Eqs (9) and taking into account that $\sin z_{1}^{\prime}(t)=z_{1}^{\prime}(t)-(1 / 3 !) z_{1}^{\prime 3}(t)+(1 / 5 !) z_{1}^{\prime 5}(t)-\cdots$, the mathematical model of the pendulum with harmonic oscillations in the axes OX and OY and with PID control can be written in matrix form (up to third order terms) as follows:

$$
\begin{gathered}
{\left[\begin{array}{c}
\dot{z}_{1}^{\prime}(t) \\
\dot{z}_{2}^{\prime}(t) \\
\dot{z}_{3}^{\prime}(t)
\end{array}\right]=\left[\begin{array}{ccc}
0 & 1 & 0 \\
\omega_{0}^{2} & -\delta & \alpha \\
-K_{p}\left(1 / \tau_{i}+\tau_{d} \omega_{0}^{2}\right) & -K_{p}\left(1-\tau_{d} \delta\right) & -\alpha K_{p} \tau_{d}
\end{array}\right]\left[\begin{array}{c}
z_{1}^{\prime}(t) \\
z_{2}^{\prime}(t) \\
z_{3}^{\prime}(t)
\end{array}\right]+} \\
+\left[\begin{array}{c}
0 \\
-\left(\omega_{0}^{2} / 3 !\right) \dot{z}_{1}^{\prime 3}(t)+\cdots \\
\left(\omega_{0}^{2} K_{p} \tau_{d} / 3 !\right) \dot{z}_{1}^{\prime 3}(t)+\cdots
\end{array}\right]+\left[\begin{array}{c}
0 \\
-(1 / l)\left[-\ddot{x}_{0}(t) \cos z_{1}^{\prime}(t)-\ddot{y}_{0}(t) \sin z_{1}^{\prime}(t)\right] \\
\left(K_{p} \tau_{d} / l\right)\left[-\ddot{x}_{0}(t) \cos z_{1}^{\prime}(t)-\ddot{y}_{0}(t) \sin z_{1}^{\prime}(t)\right]
\end{array}\right]
\end{gathered}
$$

The eigenvalues of the matrix A associated to the linear part of Eq (20) can be obtained from the Routh criterion [39], and in addition we can investigate admissible values for the parameters $K_{p}, \tau_{i}$ and $\tau_{d}$ of the PID controller. Since the system is of third order, we pretend to obtain one real negative and two pure imaginary eigenvalues, so a weak focus appears around $\theta=\pi$ [34-37]. If such weak focus is stable, the pendulum can be maintained around the pointing-up position with smooth oscillations. The eigenvalues are obtained as the roots of the characteristic equation of matrix A, i.e.: 


$$
\begin{aligned}
& |\lambda I-A|=\lambda^{3}+\left(\delta+\alpha K_{p} \tau_{d}\right) \lambda^{2}+\left(\alpha K_{p}-\omega_{0}^{2}\right) \lambda+\alpha K_{p} / \tau_{i} \\
& =\lambda^{3}+a_{2} \lambda^{2}+a_{1} \lambda+a_{0}=0
\end{aligned}
$$

where the following conditions must be verified:

$$
a_{1}>0 \Rightarrow \alpha K_{p}-\alpha ; a_{1} a_{2}-a_{0}=0 \Rightarrow\left(\delta+\alpha K_{p} \tau_{d}\right)\left(\alpha K_{p}-\omega_{0}^{2}\right)=\alpha K_{p} / \tau_{i}
$$

The self-oscillation frequency is given by:

$$
\begin{gathered}
\left(\delta+\alpha K_{p} \tau_{d}\right) \lambda^{2}+\alpha K_{p} / \tau_{i}=0 \Rightarrow \lambda_{1,-1}= \pm \omega i \\
\omega=\sqrt{\frac{\alpha K_{p}}{\tau_{i}\left(\delta+\alpha K_{p} \tau_{d}\right)}}
\end{gathered}
$$

and from Eqs (22) and (23) it is deduced that:

$$
\alpha K_{p}-\omega_{0}^{2}=\frac{\alpha K_{p}}{\tau_{i}\left(\delta+\alpha K_{p} \tau_{d}\right)}=\omega^{2} \Rightarrow \alpha K_{p}-\omega_{0}^{2}>0
$$

Consequently, if the PID parameters are chosen in accordance with Eq (22), the inequality $a_{1}>0$ is fulfilled and the roots of the characteristic equation are $\lambda_{0}=-\left(\delta+\alpha K_{p} \tau_{d}\right)$ and $\lambda_{1,-1}= \pm i \omega$ (which can be verified substituting $\lambda_{\mathrm{i}}$ for $\mathrm{i}=0,1,-1$ into Eq (21)). Therefore, in the unstable pointing-up position we have two pure imaginary eigenvalues as well as a real negative one, whose stability will be analyzed in the next section.

\section{Stability analysis by using the normal form theory}

In accordance with the results of the previous section, we shall determine the stability conditions for the weak focus as a function of the parameters of the control law given by Eq (8). The stability analysis is carried out by using the normal form theory proposed by Bruno [27-31], since it provides a direct connection between the original and transformed equations of the system. The first step consists of obtaining the (complex) Jordan canonical form of Eqs (20) taking only up to third-order terms. The eigenvectors of the matrix given in $\mathrm{Eq}$ (20) allow to find the matrix $\mathrm{P}$ associated to a 
linear transformation which transforms the matrix of the linear part into its complex Jordan canonical form, i.e.:

$$
\begin{gathered}
z^{\prime}(t)=P x(t) \Rightarrow\left[\begin{array}{c}
z_{1}^{\prime}(t) \\
z_{2}^{\prime}(t) \\
z_{3}^{\prime}(t)
\end{array}\right]=\left[\begin{array}{ccc}
1 & 1 & 1 \\
+i \omega & -i \omega & -\left(\delta+\alpha K_{p} \tau_{d}\right) \\
p_{31} & p_{32} & p_{33}
\end{array}\right]\left[\begin{array}{c}
x_{1}(t) \\
x_{-1}(t) \\
x_{0}(t)
\end{array}\right] \\
p_{31}=-K_{p}+\left(\frac{K_{p}}{\tau_{i} \omega}-\omega K_{p} \tau_{d}\right) i ; p_{32}=-K_{p}-\left(\frac{K_{p}}{\tau_{i} \omega}-\omega K_{p} \tau_{d}\right) i \\
p_{33}=\frac{1}{\alpha}\left[-\omega_{0}^{2}+\alpha K_{p} \tau_{d}\left(\delta+\alpha K_{p} \tau_{d}\right)\right]
\end{gathered}
$$

Taking into account Eqs (25), Eqs (20) are transformed as follows:

$$
\begin{array}{r}
{\left[\begin{array}{c}
\dot{x}_{1}(t) \\
\dot{x}_{-1}(t) \\
\dot{x}_{0}(t)
\end{array}\right]=\left[\begin{array}{ccc}
i \omega & 0 & 0 \\
0 & -i \omega & 0 \\
0 & 0 & \delta_{e}
\end{array}\right]\left[\begin{array}{c}
\dot{x}_{1}(t) \\
\dot{x}_{-1}(t) \\
\dot{x}_{0}(t)
\end{array}\right]+P^{-1}\left[\begin{array}{c}
0 \\
-\left(\omega_{0}^{2} / 3 !\right)\left[x_{1}(t)+x_{-1}(t)+x_{0}(t)\right]^{3} \\
\left(\omega_{0}^{2} K_{p} \tau_{d} / 3 !\right)\left[x_{1}(t)+x_{-1}(t)+x_{0}(t)\right]^{3}
\end{array}\right]} \\
+P^{-1}\left[\begin{array}{c}
0 \\
-(1 / l)\left[-\ddot{x}_{0}(t) \cos \left\{\left[x_{1}(t)+x_{-1}(t)+x_{0}(t)\right]\right\}-\ddot{y}_{0}(t) \sin \left\{\left[x_{1}(t)+x_{-1}(t)+x_{0}(t)\right]\right\}\right] \\
\left(K_{p} \tau_{d} / l\right)\left[-\ddot{x}_{0}(t) \cos \left\{\left[x_{1}(t)+x_{-1}(t)+x_{0}(t)\right]\right\}-\ddot{y}_{0}(t) \sin \right]\left\{\left[x_{1}(t)+x_{-1}(t)+x_{0}(t)\right]\right\}
\end{array}\right]
\end{array}
$$

where $\delta_{e}=\delta+\alpha K_{p} \tau_{d}$ is an equivalent damping coefficient and $\mathrm{P}^{-1}$ denotes the inverse matrix of $\mathrm{P}$, which is given by:

$$
\begin{aligned}
P^{-1} & =\frac{1}{\Delta}\left[\begin{array}{ccc}
p_{11}^{-1} & p_{12}^{-1} & -\delta_{e}+\omega i \\
p_{21}^{-1} & p_{22}^{-1} & \delta_{e}+\omega i \\
-2 \omega K_{p} i & \left(\left(K_{p} / \tau_{i} \omega\right)-\omega K_{p} \tau_{d}\right) i & -2 \omega i
\end{array}\right] \\
\Delta & =2\left[\omega\left(\frac{\omega_{0}^{2}}{\alpha}-K_{p} \delta_{e} \tau_{d}\right)-K_{p} \omega-\delta_{e}\left(\frac{K_{p}}{\tau_{i} \omega}-\omega K_{p} \tau_{d}\right)\right] i \\
p_{11}^{-1}= & \left.=\omega\left(\frac{\omega_{0}^{2}}{\alpha}-K_{p} \delta_{e} \tau_{d}\right) i+\delta_{e}\left[-K_{p}-\left(\frac{K_{p}}{\tau_{i} \omega}-\omega K_{p} \tau_{d}\right)\right] i\right] \\
p_{21}^{-1} & =\omega\left(\frac{\omega_{0}^{2}}{\alpha}-K_{p} \delta_{e} \tau_{d}-K_{p}-\left(\frac{K_{p}}{\tau_{i} \omega}-\omega K_{p} \tau_{d}\right) i-\delta_{e}\left[-K_{p 2}^{-1}=-\frac{\omega_{0}^{2}}{\alpha}+K_{p} \delta_{e} \tau_{d}+K_{p}-\left(\frac{K_{p}}{\tau_{i} \omega}-\omega K_{p} \tau_{d}\right) i\right]\right.
\end{aligned}
$$


Assuming that there are no disturbances at the supporting point $\mathrm{O}^{\prime}$ of the pendulum (see Fig 1), the system defined by Eq (26) has the following general form:

$$
\frac{d x_{v}}{d t}=\lambda_{v} x_{v}+\sum b_{l m p}^{v} x_{l} x_{m} x_{p} ; v=0, \pm 1 ; l, m, p=0, \pm 1
$$

where the eigenvalues of the linear part are $\lambda_{0}=-\left(\delta+\alpha K_{p} \tau_{d}\right), \lambda_{1}=i \omega$ and $\lambda_{-1}=-i \omega$. The coefficients $b_{l m p}^{v}$ can in general be complex and they are invariant under permutations of sub-indices $1, \mathrm{~m}$ and $\mathrm{p}$. Once the complex Jordan canonical form has been obtained, the idea of the formal norm theory is to obtain a simplified version of the original system which retains all its dynamical properties [17-18], [32]. The normal form is based on a new change of variables called reversible normalizing transformation, which is defined as:

$$
x_{v}=y_{v}+\sum \beta_{l m p}^{v} y_{l} y_{m} y_{p} ; v=0, \pm 1 ; l, m, p=0, \pm 1
$$

Equation (29) considers only terms up to third order and allows to transform the system given by Eq (27) into its normal form, which is defined as:

$$
\frac{d y_{v}}{d t}=\lambda_{v} y_{v}+y_{v} \sum_{[\Lambda, Q]=0} g_{v Q} y_{0}^{q_{0}} y_{1}^{q_{1}} y_{-1}^{q_{-1}} ; v=0, \pm 1
$$

being $\mathrm{q}_{0}, \mathrm{q}_{1}$ and $\mathrm{q}_{-1}$ integer numbers that must satisfy the following relations [27-28]:

$$
\begin{gathered}
\Lambda=\left[\begin{array}{ccc}
\lambda_{0} & \lambda_{1} & \lambda_{-1}
\end{array}\right] ; Q=\left[\begin{array}{lll}
q_{0} & q_{1} & q_{-1}
\end{array}\right] ;[\Lambda, Q]=\lambda_{0} q_{0}+\lambda_{1} q_{1}+\lambda_{-1} q_{-1}=0 \\
q_{v} \geq-1 ; q_{j} \geq 0(j \neq v)(v=0, \pm 1) ; q_{0}+q_{1}+q_{-1} \geq 1
\end{gathered}
$$

Taking into account Eqs (31) and (32), the relation between the coefficients $b_{l m p}^{v}$ and $\beta_{l m p}^{v}$ can be expressed as follows:

$$
\beta_{l m p}^{v}=\left\{\begin{array}{c}
\frac{b_{l m p}^{v}}{\lambda_{l}+\lambda_{m}+\lambda_{p}-\lambda_{v}} \text { if } \lambda_{l}+\lambda_{m}+\lambda_{p}-\lambda_{v} \neq 0 \\
0 \quad \text { if } \lambda_{l}+\lambda_{m}+\lambda_{p}-\lambda_{v}=0
\end{array}\right\} v=0, \pm 1 ; l, m, p=0, \pm 1
$$


Equation (30) can be written in similar way as Eqs (28), i.e.:

$$
\frac{d y_{v}}{d t}=\lambda_{v} x_{v}+\sum \chi_{l m p}^{v} y_{l} y_{m} y_{p} ; v=0, \pm 1 ; l, m, p=0, \pm 1
$$

where the only non-null coefficients are the ones of the form $\chi_{\{v, 1,-1\}}^{v}($ where $\{v, 1-1\}$ denote any permutation of the elements $v, 1$ and -1$)$, since $\beta_{\{v, 1,-1\}}^{v}=0 ; v=0, \pm 1$ as it follows from Eqs (31)-(33). From the previous considerations, Eqs (30) and (34) can be expanded as follows [27-28] [32]:

$$
\left.\left.\begin{array}{l}
\frac{d y_{1}}{d t}=i \omega y_{1}+y_{1} g_{1}^{1} y_{1} y_{-1} \\
\frac{d y_{-1}}{d t}=-i \omega y_{-1}+y_{-1} g_{1}^{-1} y_{1} y_{-1} \\
\frac{d y_{0}}{d t}=-\delta_{e} y_{0}+y_{0} g_{1}^{0} y_{1} y_{-1}
\end{array}\right\} \begin{array}{c}
\frac{d y_{1}}{d t}=i \omega y_{1}+\chi_{\{1,1,-1\}}^{1} y_{1} y_{1} y_{-1} \\
\frac{d y_{-1}}{d t}=-i \omega y_{-1}+\chi_{\{1,1,-1\}}^{-1} y_{-1} y_{1} y_{-1} \\
\frac{d y_{0}}{d t}=-\delta_{e} y_{0}+\chi_{\{1,1,-1\}}^{0} y_{0} y_{1} y_{-1}
\end{array}\right\}
$$

From Eqs (33) and (35) it is deduced that:

$$
g_{1}^{1}=3 \chi_{11-1}^{1}=3 b_{11-1}^{1} \quad ; \quad g_{1}^{-1}=\left(g_{1}^{1}\right)^{*} ; g_{1}^{0}=6 \chi_{01-1}^{1}=6 b_{01-1}^{0}
$$

where the asterisk denotes conjugate complex. To calculate the normalizing transformation given by Eqs (29), Eq (33) must be applied taking into account that the coefficients $b_{l m p}^{v}$ are deduced by identifying terms between Eqs (26)-(28). For this purpose, the following cases must be considered:

- Case $v=1$

In this case all the coefficients $b_{l m p}^{v}$ take the form:

$$
b_{11-1}^{1}=b^{1}=\frac{\omega_{0}^{2}}{2.3 !} \frac{\frac{K_{p}}{\tau_{i} \omega}+\left(\frac{\omega_{0}^{2}}{\alpha}-K_{p}\right) i}{\omega\left(\frac{\omega_{0}^{2}}{\alpha}-\delta_{e} K_{p} \tau_{d}\right)-K_{p} \omega-\delta_{e}\left(\frac{K_{p}}{\tau_{i} \omega}-\delta_{e} K_{p} \tau_{d}\right)}
$$


and from Eq (33) the successive values of $\beta_{l m p}^{v}$ are obtained as:

$$
\begin{gathered}
\beta_{000}^{1}=\frac{b_{000}^{1}}{\lambda_{0}+\lambda_{0}+\lambda_{0}-\lambda_{1}}=-\frac{b^{1}}{3 \delta_{e}+\omega i} ; \beta_{111}^{1}=\frac{b_{111}^{1}}{\lambda_{1}+\lambda_{1}+\lambda_{1}-\lambda_{1}}=\frac{b^{1}}{2 \omega i} \\
\beta_{-1-1-1}^{1}=\frac{b_{-1-1-1}^{1}}{\lambda_{0}+\lambda_{0}+\lambda_{0}-\lambda_{1}}=-\frac{b^{1}}{4 \omega i} ; \beta_{001}^{1}=\frac{b_{001}^{1}}{\lambda_{0}+\lambda_{0}+\lambda_{1}-\lambda_{1}}=-\frac{b^{1}}{2 \delta_{e}} \\
\beta_{00-1}^{1}=\frac{b_{00-1}^{1}}{\lambda_{0}+\lambda_{0}+\lambda_{-1}-\lambda_{1}}=-\frac{b^{1}}{2 \delta_{e}+2 \omega i} ; \beta_{011}^{1}=\frac{b_{011}^{1}}{\lambda_{0}+\lambda_{1}+\lambda_{1}-\lambda_{1}}=\frac{b^{1}}{-\delta_{e}+\omega i} \\
\beta_{0-1-1}^{1}=\frac{b_{0-1-1}^{1}}{\lambda_{0}+\lambda_{-1}+\lambda_{-1}-\lambda_{1}}=\frac{b^{1}}{-\delta_{e}-3 \omega i} ; \beta_{1-1-1}^{1}=\frac{b_{001}^{1}}{\lambda_{1}+\lambda_{-1}+\lambda_{-1}-\lambda_{1}}=-\frac{b^{1}}{2 \omega i} \\
\beta_{01-1}^{1}=\frac{b_{01-1}^{1}}{\lambda_{0}+\lambda_{1}+\lambda_{-1}-\lambda_{1}}=-\frac{b^{1}}{\delta_{e}+\omega i}
\end{gathered}
$$

- $\quad$ Case $v=0$

In this case all the coefficients $b_{l m p}^{v}$ take the form:

$$
b_{11-1}^{0}=b^{0}=\frac{\omega_{0}^{2}}{3 !} \frac{\frac{K_{p}}{\tau_{i} \omega}}{\omega\left(\frac{\omega_{0}^{2}}{\alpha}-\delta_{e} K_{p} \tau_{d}\right)-K_{p} \omega-\delta_{e}\left(\frac{K_{p}}{\tau_{i} \omega}-\delta_{e} K_{p} \tau_{d}\right)}
$$

and Eq (33) allows to obtain the successive values of $\beta_{l m p}^{v}$ as follows:

$$
\begin{gathered}
\beta_{000}^{0}=\frac{b_{000}^{0}}{\lambda_{0}+\lambda_{0}+\lambda_{0}-\lambda_{0}}=-\frac{b^{0}}{2 \delta_{e}} ; \beta_{111}^{0}=\frac{b_{111}^{0}}{\lambda_{1}+\lambda_{1}+\lambda_{1}-\lambda_{0}}=\frac{b^{0}}{\delta_{e}+2 \omega i} \\
\beta_{-1-1-1}^{0}=\frac{b_{-1-1-1}^{0}}{\lambda_{0}+\lambda_{0}+\lambda_{0}-\lambda_{0}}=\frac{b^{0}}{\delta_{e}-3 \omega i} ; \beta_{001}^{0}=\frac{b_{001}^{0}}{\lambda_{0}+\lambda_{0}+\lambda_{1}-\lambda_{0}}=-\frac{b^{0}}{-\delta_{e}+\omega i} \\
\beta_{00-1}^{0}=\frac{b_{00-1}^{0}}{\lambda_{0}+\lambda_{0}+\lambda_{-1}-\lambda_{0}}=-\frac{b^{0}}{\delta_{e}+\omega i} ; \beta_{011}^{0}=\frac{b_{011}^{0}}{\lambda_{0}+\lambda_{1}+\lambda_{1}-\lambda_{0}}=\frac{b^{0}}{2 \omega i} \\
\beta_{0-1-1}^{0}=\frac{b_{0-1-1}^{0}}{\lambda_{0}+\lambda_{-1}+\lambda_{-1}-\lambda_{0}}=-\frac{b^{0}}{2 \omega i} ; \beta_{1-1-1}^{0}=\frac{b_{001}^{0}}{\lambda_{1}+\lambda_{-1}+\lambda_{-1}-\lambda_{1}}=\frac{b^{0}}{\delta_{e}-\omega i} \\
\beta_{01-1}^{0}=\frac{b_{01-1}^{0}}{\lambda_{0}+\lambda_{1}+\lambda_{-1}-\lambda_{0}} ; \lambda_{0}+\lambda_{1}+\lambda_{-1}-\lambda_{0}=0 \Rightarrow \beta_{01-1}^{0}=0
\end{gathered}
$$

Taking into account Eqs (29), (38) and (40), the normalizing transformation of the variables $\mathrm{x}_{1}$ and $\mathrm{x}_{0}$ can be written as: 


$$
\begin{gathered}
x_{1}=y_{1}+b^{1}\left[\begin{array}{c}
\frac{-y_{0}^{3}}{3 \delta_{e}+\omega i}+\frac{y_{1}^{3}}{2 \omega i}-\frac{y_{-1}^{3}}{4 \omega i}-\frac{3 y_{0}^{2} y_{1}}{2 \delta_{e}}-\frac{3 y_{0}^{2} y_{-1}}{2 \delta_{e}+2 \omega i} \\
+\frac{3 y_{0} y_{1}^{2}}{-\delta_{e}+\omega i}-\frac{3 y_{0} y_{-1}^{2}}{\delta_{e}+3 \omega i}-\frac{3 y_{1} y_{-1}^{2}}{2 \omega i}-\frac{6 y_{0} y_{1} y_{-1}}{\delta_{e}+\omega i}
\end{array}\right] \\
x_{0}=y_{0}+b^{0}\left[\begin{array}{c}
-\frac{y_{0}^{3}}{2 \delta_{e}}+\operatorname{Re}\left(\frac{y_{1}^{3}}{\delta_{e}+3 \omega i}\right)+\operatorname{Re}\left(\frac{y_{-1}^{3}}{\delta_{e}-3 \omega i}\right)+3 y_{0}^{2} \operatorname{Re}\left(\frac{y_{1}}{-\delta_{e}+\omega i}\right) \\
+3 y_{0}^{2} \operatorname{Re}\left(\frac{y_{1}}{-\delta_{e}-\omega i}\right)+3 y_{0} \operatorname{Re}\left(\frac{y_{1}^{2}}{2 \omega i}\right)+3 y_{0} \operatorname{Re}\left(\frac{y_{1}^{2}}{-2 \omega i}\right) \\
+3 y_{1} y_{-1} \operatorname{Re}\left(\frac{y_{-1}}{\delta_{e}-\omega i}\right)
\end{array}\right]
\end{gathered}
$$

Since $\mathrm{x}_{0}$ and $\mathrm{y}_{0}$ are real whereas $\mathrm{y}_{1}$ and $\mathrm{y}_{-1}$ are conjugate complex, the appropriate real part of the terms $b_{0} y_{l} y_{m} y_{p}$ must be considered to obtain real coefficients in Eq. (42). By adding the terms that have the same power of $y_{0}$ and $y_{1}, E q(42)$ can be rewritten as follows:

$$
x_{0}=y_{0}+b^{0}\left[\begin{array}{c}
-\frac{y_{0}^{3}}{2 \delta_{e}}+2 \operatorname{Re}\left(\frac{y_{1}^{3}}{\delta_{e}+3 \omega i}\right)+6 y_{0}^{2} \operatorname{Re}\left(\frac{y_{1}}{-\delta_{e}+\omega i}\right) \\
+6 y_{0} \operatorname{Re}\left(\frac{y_{1}^{2}}{2 \omega i}\right)+3 y_{1} y_{-1} \operatorname{Re}\left(\frac{y_{-1}}{\delta_{e}-\omega i}\right)
\end{array}\right]
$$

The normalizing transformations given by Eqs (41) and (43) have been deduced up to third-order terms. The inverse transformation (also up to third-order terms) can be obtained by removing the variables $\mathrm{y}_{0}$ and $\mathrm{y}_{1}$ from the right hand sides of Eqs (41) and (43) and replacing such variables by $\mathrm{x}_{0}$ and $\mathrm{x}_{1}$ inside the braces [18], which leads to:

$$
y_{1}=x_{1}-b^{1}\left[\begin{array}{c}
\frac{-x_{0}^{3}}{3 \delta_{e}+\omega i}+\frac{x_{1}^{3}}{2 \omega i}-\frac{x_{-1}^{3}}{4 \omega i}-\frac{3 x_{0}^{2} x_{1}}{2 \delta_{e}}-\frac{3 x_{0}^{2} x_{-1}}{2 \delta_{e}+2 \omega i} \\
+\frac{3 x_{0} x_{1}^{2}}{-\delta_{e}+\omega i}-\frac{3 x_{0} x_{-1}^{2}}{\delta_{e}+3 \omega i}-\frac{3 x_{1} x_{-1}^{2}}{2 \omega i}-\frac{6 x_{0} x_{1} x_{-1}}{\delta_{e}+\omega i}+\mathrm{O}\left\|x_{i}^{4}\right\|
\end{array}\right]
$$




$$
y_{0}=x_{0}-b^{0}\left[\begin{array}{c}
-\frac{x_{0}^{3}}{2 \delta_{e}}+2 \operatorname{Re}\left(\frac{x_{1}^{3}}{\delta_{e}+3 \omega i}\right)+6 y_{0}^{2} \operatorname{Re}\left(\frac{x_{1}}{-\delta_{e}+\omega i}\right) \\
6 x_{0} \operatorname{Re}\left(\frac{x_{1}^{2}}{2 \omega i}\right)+3 x_{1} x_{-1} \operatorname{Re}\left(\frac{x_{-1}}{\delta_{e}-\omega i}\right)+\mathrm{O}\left\|x_{i}^{4}\right\|
\end{array}\right]
$$

It should be noted that the numerical solution of the problem given by Eqs (20) can be obtained by removing the harmonic disturbances and considering all the terms of the Taylor series for the sinus function. Then, the variables $x_{1}(t), x_{-1}(t)$ and $x_{0}(t)$ can be obtained by means of Eqs (25) and (27), and an approximate solution for the system in normal form can be deduced from Eqs (44) and (45).

The interest of the system equations in normal form relies on the fact that they allow to deduce an exact analytical solution from Eqs (35). For this purpose, multiplying by $\mathrm{y}_{-1}(\mathrm{t})$ the first equation of (35) and by $\mathrm{y}_{1}(\mathrm{t})$ the second one, taking into account that $g_{1}^{1}+g_{1}^{-1}=2 \operatorname{Re}\left(g_{1}^{1}\right)$ and introducing the variable $p_{1}(t)=y_{1 a}(t) y_{-1 a}(t)$, the following differential equation can be deduced:

$$
\frac{d p_{1}(t)}{d t}=p_{1}^{2}(t)+2 \operatorname{Re}\left[g_{1}^{1}\right] \Rightarrow p_{1}(t)=\frac{\left|y_{1 a}(0)\right|^{2}}{1-2 \operatorname{Re}\left[g_{1}^{1}\right] t} ; p_{1}(0)=\left|y_{1 a}(0)\right|^{2}
$$

where the sub-index "a" has been introduced to distinguish the analytical solution from the normal form, which has been deduced from the inverse normalizing transformation given by Eqs (44)-(45). If we assume that $2 \operatorname{Re}\left[g_{1}^{1}\right]>0$ for $t \rightarrow \infty, \mathrm{Eq}(46)$ allows to deduce that the variable $p_{l}(t)$ will eventually be negative, which is impossible since $p_{1}(t)=y_{1 a}(t) y_{-1 a}(t)=\left|y_{1 a}(t)\right|^{2}$. On the other hand, if $2 \operatorname{Re}\left[g_{1}^{1}\right]<0$ for $t \rightarrow \infty$ then $p_{1}(t) \rightarrow 0$ and therefore the system will be stable. Consequently, taking into account the equations of the system in normal form as well as Eqs (36)-(37), the stability condition for the weak focus associated to the pointing-up position of the pendulum can be written as follows: 


$$
\operatorname{Re}\left[g_{1}^{1}\right]=\operatorname{Re}\left[3 b^{1}\right]=\frac{\omega_{0}^{2}}{2.3 !} \frac{3 \frac{K_{p}}{\tau_{i} \omega}}{\omega\left(\frac{\omega_{0}^{2}}{\alpha}-\delta_{e} K_{p} \tau_{d}\right)-K_{p} \omega-\delta_{e}\left(\frac{K_{p}}{\tau_{i} \omega}-\delta_{e} K_{p} \omega\right)}<0
$$

The inequality given in $\mathrm{Eq}(47)$ is verified if:

$$
\alpha K_{p}>\frac{\tau_{i} \omega^{2}}{\tau_{i} \omega^{2}+\delta_{e}} \omega_{0}^{2}
$$

It should be noticed that the inequality (48) is always verified, since starting from the hypothesis that there is a weak focus (see Eq (24)) we obtain that $\alpha K_{p}=\omega_{0}^{2}+\omega^{2}$. Consequently, the pendulum in the equilibrium point $\theta_{\mathrm{e}}=\pi$ is stable.

The exact solution for the normal form can be obtained as follows. Taking into account that $p_{1}(t)=y_{1 a}(t) y_{-1 a}(t)$, from the first equation of (35) it is deduced that:

$$
\frac{d y_{1 a}(t)}{y_{1 a}(t)}=\left[\omega i+g_{1}^{1} p_{1}(t)\right] d t \Rightarrow y_{1 a}(t)=y_{1 a}(0) \exp \left[i \omega t+\int_{0}^{t} g_{1}^{1} p_{1}(\tau) d \tau\right]
$$

From Eqs (46) and (49), the variable $y_{l}(t)$ can be written as:

$$
y_{1 a}(t)=y_{1 a}(0) \exp (i \omega t)\left[1-2\left|y_{1 a}(0)\right|^{2} \operatorname{Re}\left[g_{1}^{1}\right] t\right]^{-\frac{g_{1}^{1}}{2 \operatorname{Re}\left[g_{1}^{1}\right]}}
$$

where

$$
\begin{gathered}
-\frac{g_{1}^{1}}{2 \operatorname{Re}\left[g_{1}^{1}\right]}=-\frac{1}{2} \frac{K_{p} / \tau_{i} \omega+\left[\left(\omega_{0}^{2} / \alpha\right)-K_{p}\right]}{K_{p} / \tau_{i} \omega}=-\frac{1}{2}+b^{2} i \\
b^{2}=-\frac{1}{2} \frac{\left[\left(\omega_{0}^{2} / \alpha\right)-K_{p}\right]}{K_{p} / \tau_{i} \omega}>0
\end{gathered}
$$

Substituting Eq (51) into Eq (50) we obtain that: 


$$
\begin{gathered}
y_{1 a}(t)=\frac{y_{1 a}(0)}{\sqrt{1+a^{2}\left|y_{1 a}(0)\right|^{2} t}} \exp i\left\{\omega t+b^{2} \ln \left[1+a^{2}\left|y_{1 a}(0)\right|^{2} t\right]\right\} \\
a^{2}=-2 \operatorname{Re}\left[g_{1}^{1}\right]>0
\end{gathered}
$$

where $y_{-1 a}(t)$ is the conjugate complex of $y_{1 a}(t)$. Following a similar procedure, the value of $\mathrm{y}_{0}(\mathrm{t})$ can be obtained as [17-18]:

$$
y_{0 a}(t)=y_{0 a}(0) \exp \left(-\delta_{e} t\right)\left[1-2\left|y_{1 a}(0)\right|^{2} \operatorname{Re}\left[g_{1}^{1}\right] t\right]^{-\frac{g_{1}^{0}}{2 \operatorname{Re}\left[g_{1}^{1}\right]}} ;-\frac{g_{1}^{0}}{2 \operatorname{Re}\left[g_{1}^{1}\right]}=2
$$

Consequently, Eqs (52) and (53) provide the general analytic solution for the normal form (see Eq (35)) with accuracy up to third-order terms. The stability consideration deduced from the normal form can also be used to adjust the parameters $K_{p}, \tau_{i}$ and $\tau_{d}$ of the PID controller throughout the following steps:

1. From the condition for obtaining a stable weak focus (see Eqs (22)-(24)) it is deduced that:

$$
\alpha K_{p}=\omega^{2}+\omega_{0}^{2}=\frac{\alpha K_{p}}{\delta_{e} \tau_{i}}+\omega_{0}^{2} \Rightarrow \alpha K_{p}\left(1-\frac{1}{\delta_{e} \tau_{i}}\right)=\omega_{0}^{2} \Rightarrow \delta_{e} \tau_{i}>1
$$

In accordance with the inequality given by $\mathrm{Eq}(54)$, we take $\delta_{e} \tau_{i}=f ; f>1$. Once the factor $f$ has been chosen, the value of $K_{p}$ will be given by:

$$
K_{p}=\frac{f \omega_{0}^{2}}{\alpha(f-1)}
$$

2. Next we choose a value for $\tau_{\mathrm{d}}$ to obtain an appropriate value for the equivalent damping coefficient given by $\delta_{e}=\delta+\alpha K_{p} \tau_{d}=b / m l^{2}+\alpha K_{p} \tau_{d}$. If b $\ll 1$ then $\tau_{\mathrm{d}}$ must be large, and on the contrary, $\tau_{\mathrm{d}}$ must be small if $\mathrm{b} \approx 1$. 
3. Once the values for $K_{p}$ and $\tau_{d}$ are known, the reset time $\tau_{i}$ of the PID controller is given by:

$$
\tau_{i}=\frac{f}{\delta_{e}}=\frac{f}{\delta+\alpha K_{p} \tau_{d}}
$$

After obtaining the normalizing transformations and the parameters of the PID controller, we shall analyze the relationship between the variables $z_{i}{ }_{i}(t)(i=1,2,3), x_{1}(t)$, $\mathrm{x}_{-1}(\mathrm{t}), \mathrm{x}_{0}(\mathrm{t})$ and $\mathrm{y}_{1}(\mathrm{t}), \mathrm{y}_{-1}(\mathrm{t}), \mathrm{y}_{0}(\mathrm{t})$. The values of $\mathrm{z}_{\mathrm{i}}(\mathrm{t})$ can be obtained through the simulation of Eqs (9) and (19). On the other hand, the variables $x_{1}(t), x_{-1}(t)$ and $x_{0}(t)$ can be obtained as functions of $z_{i}^{\prime}(t)(i=1,2,3)$ from the inverse normalizing transformation, which is deduced from Eqs (25), (27), (41) and (42), i.e.:

$$
\begin{gathered}
\left\{\omega\left(\frac{\omega_{0}^{2}}{\alpha}-K_{p} \delta_{e} \tau_{d}\right) i-\delta_{e}\left[-K_{p}+\left(\frac{K_{p}}{\tau_{i} \omega}-\omega K_{p} \tau_{d}\right) i\right]\right\} z_{1}^{\prime}(t) \\
\left.+\left\{-\frac{\omega_{0}^{2}}{\alpha}+K_{p} \delta_{e} \tau_{d}+K_{p}-\left(\frac{K_{p}}{\tau_{i} \omega}-\omega K_{p} \tau_{d}\right) i\right\} z_{2}^{\prime}(t)+\left(\delta_{e}+\omega i\right) z_{3}^{\prime}(t)\right\} \\
x_{1}(t)=\frac{2\left[\omega\left(\frac{\omega_{0}^{2}}{\alpha}-K_{p} \delta_{e} \tau_{d}\right)-K_{p} \omega-\delta_{e}\left(\frac{K_{p}}{\tau_{i} \omega}-\omega K_{p} \tau_{d}\right)\right] i}{2\left[\omega\left(\frac{\omega_{0}^{2}}{\alpha}-K_{p} \delta_{e} \tau_{d}\right)-K_{p} \omega-\delta_{e}\left(\frac{K_{p}}{\tau_{i} \omega}-\omega K_{p} \tau_{d}\right)\right] i} \\
x_{0}(t)=\frac{\left[-2 \omega K_{p} i\right] z_{1}^{\prime}(t)+\left[2\left(\left(K_{p} / \tau_{i} \omega\right)-\omega K_{p} \tau_{d}\right) i\right] z_{2}^{\prime}(t)+[-2 \omega i] z_{3}^{\prime}(t)}{}
\end{gathered}
$$

where $\mathrm{x}_{-1}(\mathrm{t})$ is the conjugate complex of $\mathrm{x}_{1}(\mathrm{t})$. Taking into account Eqs (44) and (45), the variables of the system in normal form $\mathrm{y}_{1}(\mathrm{t}), \mathrm{y}_{-1}(\mathrm{t})$ and $\mathrm{y}_{0}(\mathrm{t})$ can be obtained and can be compared with Eqs (52) and (53). Consequently, we have deduced a procedure to compare the analytical derivations with the numerical simulations for the considered parameters of the PID controller given by Eqs (54)-(56).

The parameters of the PID controller have been chosen according to Eqs (54)(56) assuming $f=2$ to obtain small values for $b^{1}$ and $b^{0}$ (Eqs (37) and (39)), so the values for $\mathrm{y}_{1}(\mathrm{t})$ and $\mathrm{y}_{0}(\mathrm{t})$ will be close to $\mathrm{x}_{1}(\mathrm{t})$ and $\mathrm{x}_{0}(\mathrm{t})$ respectively. To investigate this issue, Fig 5 a) shows the plot of the real and imaginary parts of $b^{1}$ and $b^{0}$ as a function of the values for the damping coefficient $\delta$. It should be noted that the coefficients $b^{1}$ 
and $b^{0}$ are small for large values of $\delta$. However, in accordance with Eq (56), large values for $\delta$ may lead to a very strong integral action $\left(\mathrm{K}_{\mathrm{p}} / \tau_{\mathrm{i}}\right.$ in Eq (8)) that can raise problems of saturation in the PID controller [14] [39]. For this reason, the values $m=$ $0.5 \mathrm{~kg}, 1=1 \mathrm{~m}, \mathrm{~b}=0.5 \mathrm{~N} \cdot \mathrm{m} \cdot \mathrm{s}^{2}$ and $\delta=1$ will be assumed. Fig $5 \mathrm{~b}$ ) shows the values of the reset and derivative time for $\mathrm{f}=2$, which allows to appreciate that the derivative time $\tau_{\mathrm{d}}$ must be small in comparison with the reset time $\tau_{\mathrm{i}}$ for the selected value $\delta=1$.

\section{Figure 5}

In order to apply Eqs (44), (45), (52) and (53), the initial conditions for the real and imaginary parts of $\mathrm{x}_{1}(0), \mathrm{y}_{1}(0), \mathrm{y}_{0}(\mathrm{t})$ and $\mathrm{x}_{0}(0)$ are plotted as function of the damping coefficient $\delta$ in Figs 6 a), b) and c), taking into account Eqs (57) and (58) and assuming that $z^{\prime}{ }_{1}(0)=-\pi, z_{2}^{\prime}(0)=0$ and $z_{3}{ }_{3}(0)=0$. It should be noticed that the differences between $\mathrm{x}_{\mathrm{i}}(0)$ and $\mathrm{y}_{\mathrm{i}}(0)$ are small only for large values of $\delta$, which is in accordance with the result of Fig 5 and Eqs (44)-(45). On the other hand, the phase of $\mathrm{y}_{1}(\mathrm{t})$ can be deduced from Eqs (51) and (52) as follows:

$$
F_{y_{1 a}}(t)=-\frac{1}{2} \frac{\left[\left(\omega_{0}^{2} / \alpha\right)-K_{p}\right]}{K_{p} / \tau_{i} \omega} \ln \left[1-2 \operatorname{Re}\left[g_{1}^{1}\right]\left|y_{1 a}(0)\right|^{2} t\right]
$$

Fig $6 \mathrm{~d}$ ) shows the plots for the values of $F_{y_{1} a}(t)$ as a function of the damping coefficient $\delta$ and for different values of the time t. If $F_{y_{1} a}(t)$ is close to zero or $2 \pi$, the phase difference between $\mathrm{x}_{1}(\mathrm{t})$ and $\mathrm{y}_{1 \mathrm{a}}(\mathrm{t})$ is small and thus the dynamical behavior of the PID controlled pendulum can be predicted from the analysis of the simulation results.

Figure 6

To corroborate the previous conclusions, the system has been simulated by using Eqs (9) and (19) with a damping coefficient $\delta=10$ and taking $\mathrm{f}=10$ and $\tau_{\mathrm{d}}=10^{-3} \mathrm{~s}$, which in accordance with Eqs (55) and (56) implies that $\mathrm{K}_{\mathrm{p}}=5.4444$ N.m, $\tau_{\mathrm{i}}=0.9989 \mathrm{~s}$, $\mathrm{b}^{0}=0.1614$ and $\mathrm{b}^{1}=-0.0807+0.0084 \mathrm{i}$. The variables $\theta(\mathrm{t}), \mathrm{d} \theta(\mathrm{t}) / \mathrm{dt}$ and the control torque $\mathrm{u}(\mathrm{t})$ have been plotted in Fig 7 a) showing that the pendulum remains oscillating around the pointing-up position with a small control torque. Fig 7 b) shows that the initially unstable equilibrium point $\theta_{\mathrm{e}}=\pi$ becomes a stable one, whereas Fig 7 c) shows 
that a stable weak focus appears as a result of an adequate tuning of the PID controller. On the other hand, Eqs (57) and (58) allow to deduce the values of $x_{0}(t), x_{1}(t)$ and the conjugate complex of $x_{1}(t)$ (i.e. $x_{-1}(t)$ ) once the deviation variables $z^{\prime}{ }_{1}(t) i=1,2,3$ are known through the simulation of Eqs (9) and (19). Consequently, Eqs (44)-(45) allow to calculate $\mathrm{y}_{1}(\mathrm{t})$ and $\mathrm{y}_{0}(\mathrm{t})$ and compare them with the analytical results obtained from Eqs (52)-(53), as it is shown in Fig 8. It should be noted that the values of $y_{1}(t)$ and $y_{0}(t)$ are very close to $\mathrm{y}_{1 \mathrm{a}}(\mathrm{t})$ and $\mathrm{y}_{0 \mathrm{a}}(\mathrm{t})$ respectively, in accordance with the previous considerations.

Figure 7

Figure 8

Another interesting verification of the analytical and numerical computations can be carried out taking into account the following reasoning. From Eqs (19) and (25) it is deduced that:

$$
\begin{gathered}
z_{1}^{\prime}(t)=x_{1}(t)+x_{-1}(t)+x_{0}(t) \\
z_{2}^{\prime}(t)=i \omega x_{1}(t)-i \omega x_{-1}(t)-\left(\delta+\alpha K_{p} \tau_{d}\right) x_{0}(t)
\end{gathered}
$$

Taking into account the normalizing transformations given by Eqs (42) and (43) as well as their inverse transformations given by Eqs (44) and (45), it is deduced that $x_{1}(t) \approx y_{1}(t)$ and $x_{0}(t) \approx y_{0}(t)$ as long as the coefficients $\mathrm{b}^{0}$ and $\mathrm{b}^{1}$ are small. In this case, by introducing the notation $x_{1}(0)=x_{1 r}(0)+x_{1 i}(0) i$ and taking into account Eqs (60), the squared amplitude of the pendulum in the pointing-up position and the initial condition $y_{1 a}^{2}(0)$ can be approximated as:

$$
\begin{gathered}
z_{1}^{\prime 2}(t)+z_{2}^{\prime 2}(t) \approx 4 \operatorname{Re}^{2}\left[x_{1}(t)\right]+4 \operatorname{Re}^{2}\left[i \omega x_{1}(t)\right] \\
y_{1}^{2}(0) \approx 4 \operatorname{Re}^{2}\left[x_{1}(0)\right]+4 \operatorname{Re}^{2}\left[i \omega x_{1}(0)\right]=4\left[x_{1 r}^{2}(0)+\omega^{2} x_{1 i}^{2}(0)\right]
\end{gathered}
$$

In Eqs (60) and (61) it is assumed that $x_{0}(t) \rightarrow 0$ for a sufficiently large time, which is in accordance with $\mathrm{Eq}$ (53) once the values for $\mathrm{y}_{0}(\mathrm{t})$ have been substituted by the corresponding ones for $\mathrm{x}_{0}(\mathrm{t})$. Taking into account Eqs (52) and (61), the approximate amplitude of the radius for the weak focus shown in Fig 7 c) can be calculated as follows: 


$$
A_{m a}(t)=2 \sqrt{\frac{x_{1 r}^{2}(0)+\omega^{2} x_{1 i}^{2}(0)}{1+a^{2}\left[x_{1 r}^{2}(0)+\omega^{2} x_{1 i}^{2}(0)\right]^{2} t}}
$$

The amplitude $A_{m a}(t)$ given by $\mathrm{Eq}$ (62) can be compared with the amplitude $A_{m e}(t)=y_{1}(0) / \sqrt{1+a^{2}\left|y_{1}(0)\right|^{2}} t$ which appears in Eq (52).

The previous reasoning has been corroborated in Figs 9 and 10. In Fig 9 a), the amplitudes $A_{\text {ma }}(t), A_{m e}(t)$ and the radius $r_{z}(t)$ of the weak focus have been plotted, which allows to observe that they are almost coincident. Similarly, Figs 9 b) and c) show that the attenuation of the amplitudes for $\theta(t)$ and $d \theta(t) / d t$ are very close to $A_{\text {ma }}(t)$ and $A_{\text {me }}(t)$ respectively. Fig 10 a) shows the phase of $y_{1}(t)$-deduced from Eq (59) taking into account the approximations given in $\mathrm{Eq}(61)$ - and the phase $\mathrm{F}_{\mathrm{y} 1}(\mathrm{t})$ obtained from the numerical simulations. Figs $10 \mathrm{~b}$ ), c) and d) show the values of the pendulum state variables (obtained through the simulation of Eqs (9) and (19)), which are compared with the values $\mathrm{ZZ}^{\prime}{ }_{\mathrm{i}}(\mathrm{t}) \mathrm{i}=1,2,3$ deduced from Eqs (25) and (27) by using the inverse normalizing transformations and assuming that $x_{1}(t) \approx y_{1}(t)$ and $x_{0}(t) \approx y_{0}(t)$. Once again, the validity of the analytical calculations as well as the previous hypotheses regarding the normal form have been proved.

Figure 9

Figure 10

\section{Obtaining chaotic behavior on the basis of the Melnikov function}

It is known that a pendulum with viscous damping and with an external harmonic torque applied at the suspension point can reach chaotic behavior [16-20]. On the other hand, in section 2 (see Fig 2) we deduced that the pendulum subjected to a vertical oscillation of high frequency can reach chaotic oscillations [34-38].

In this section we shall analyze the conditions to obtain chaotic behavior assuming vertical and horizontal harmonic disturbances of low frequency at the suspension point (see Fig 1). The main advantage of using low frequencies is that they require moderate forces in the OX and OY directions (which would have not been 
possible with disturbances of high frequency as shown in Fig 4 (b)). To study this issue, we consider Eq (4) assuming that the external control torque $\mathrm{u}(\mathrm{t})$ is zero. Taking into account Eqs (7), Eq (4) can be rewritten as follows:

$$
\frac{d^{2} \theta(t)}{d t^{2}}=-\frac{g}{l} \sin \theta(t)+\varepsilon\left[\begin{array}{c}
\frac{A_{x}^{\prime} \omega_{x}^{2}}{l} \sin \left(\omega_{x} t+\varphi\right) \cos \theta(t)+ \\
\frac{A_{y}^{\prime} \omega_{y}^{2}}{l} \sin \left(\omega_{y} t\right) \sin \theta(t)-\frac{b^{\prime}}{m l^{2}} \frac{d \theta(t)}{d t}
\end{array}\right]
$$

where the notation $A_{x}=\varepsilon A_{x}^{\prime} ; A_{y}=\varepsilon A_{y}^{\prime} ; b=\varepsilon b^{\prime}$ has been introduced, whereas the parameter $\varepsilon$ is a small scaling factor that has been introduced to research the conditions for chaotic dynamics. To obtain the homoclinic orbit it will be assumed that $\varepsilon=0$, for which there is no damping, the harmonic disturbances are zero and the unperturbed system is Hamiltonian. Consequently, it is possible to deduce the portrait of the phase plane by direct integration of Eqs (63). For the sake of simplifying the calculations, the following dimensionless parameters are considered:

$$
\tau=\sqrt{\frac{g}{l}} t ; \omega_{0 x}=\omega_{x} \sqrt{\frac{l}{g}} ; \gamma_{x}=\frac{A_{x}^{\prime} \omega_{x}^{2}}{g} ; \gamma_{y}=\frac{A_{y}^{\prime} \omega_{y}^{2}}{g} ; \delta=\frac{b^{\prime}}{m l^{2}} \sqrt{\frac{l}{g}}
$$

Considering the state variables $x_{1}(t)=\theta(t) ; x_{2}(t)=\dot{\theta}(t)$ as well as Eqs (63)-(64), the following equations are obtained:

$$
\begin{aligned}
& \dot{x}_{1}(\tau)=x_{2}(\tau) \\
& \dot{x}_{2}(\tau)=-\sin x_{1}(\tau)+\varepsilon\left[\gamma_{x} \sin \left(\omega_{0 x} \tau+\varphi\right) \cos x_{1}(\tau)+\gamma_{x} \sin \left(\omega_{0 y} \tau\right) \sin x_{1}(\tau)-\delta x_{2}(\tau)\right]
\end{aligned}
$$

Assuming that $\varepsilon=0$, it is deduced that the only equilibrium points are the origin -which is a centre- and the points $\pm n \pi(\mathrm{n}=1,2,3, \ldots)$-which are saddles-. Integrating Eqs (65), the parametric equations of the heteroclinic orbit are found to be:

$$
x_{1}(\tau)= \pm 2 \arcsin [\operatorname{th}(\tau)] ; x_{2}(\tau)= \pm 2 \operatorname{sech}(\tau)
$$


where the sign plus refers to an heteroclinic trajectory with $x_{4}>0$ whereas the sign minus corresponds to an heteroclinic trajectory with $x_{4}<0$. It should be remarked that currently there is not any known analytic procedure to deduce whether a nonlinear dissipative system governed by three o more differential equations is chaotic. However, Melnikov's method provides necessary conditions for chaotic dynamics (details of this method can be found in references [31-35]). Considering Eqs (65) and (66), the Melnikov's function is given by the following integral:

$$
M_{e}\left(\tau_{0}\right)=\int_{-\infty}^{+\infty} x_{2}(\tau)\left[\begin{array}{c}
\gamma_{x} \sin \left[\omega_{0 x}\left(\tau+\tau_{0}\right)+\varphi\right] \cos x_{1}(\tau)+ \\
\gamma_{y} \sin \left[\omega_{0 y}\left(\tau+\tau_{0}\right)\right] \sin x_{1}(\tau)-\delta x_{2}(\tau)
\end{array}\right] d \tau
$$

where $\tau_{0}$ is an arbitrary dimensionless time and the values of $x_{1}(\tau)$ and $x_{2}(\tau)$ must be substituted by the ones given in Eqs (66). For the purposes of this work, and taking into account standard procedures of the complex variable [34-35], the Melnikov's function can be written as [38]:

$$
M_{e}\left(\tau_{0}\right)=2 \gamma_{x} \sin \left(\omega_{0 x} \tau_{0}+\varphi\right)\left(I_{1}-I_{2}\right)+2 \gamma_{y} \cos \left(\omega_{0 x} \tau_{0}\right) I_{3}-8 \delta
$$

where:

$$
\begin{gathered}
I_{1}=\int_{-\infty}^{+\infty} \sec h(\tau) \cos \omega_{0 x} \tau d \tau=\frac{\pi}{\operatorname{ch}\left(\pi \omega_{0 x} / 2\right)} \\
I_{2}=2 \int_{-\infty}^{+\infty} \sec h(\tau) t h^{2}(\tau) \cos \omega_{0 x} \tau d \tau=\frac{\pi\left(1-\omega_{0 x}^{2}\right)}{\operatorname{ch}\left(\pi \omega_{0 x} / 2\right)} \\
I_{3}=2 \int_{-\infty}^{+\infty} \sec h(\tau) \sin \omega_{0 y} \tau \frac{\operatorname{sh}(\tau)}{\operatorname{ch}^{2}(\tau)} d \tau=\frac{\pi \omega_{0 y}^{2}}{\operatorname{sh}\left(\pi \omega_{0 y} / 2\right)}
\end{gathered}
$$

It is important to remark that a necessary condition for the occurrence of chaos is that the Melnikov's function given by Eqs (68)-(71) has non-tangential zeros. Since in our case the Melnikov's function consists of a sinusoidal term plus a constant, variations on the amplitude and/or the constant must be considered to search necessary conditions for chaotic motions. We will analyze the limit case in which $M_{0}\left(\tau_{0}\right)$ has 
tangential zeros with the ultimate purpose of bounding the sets of values for the amplitudes $A_{x}, A_{y}$ and frequencies $\omega_{x}, \omega_{y}$ that may lead to chaotic behavior. In this case, the following two possibilities can be considered:

a) Fixing a value for $\gamma_{y}$ and determining $\gamma_{x}$ so that $M_{e}\left(\tau_{0}\right)$ has no sign changes. In this case, the equation:

$$
\frac{4 \delta-2 \gamma_{y} I_{3}}{I_{1}-I_{2}}=\frac{A_{x} \omega_{x}^{2}}{g}
$$

allows to obtain $\omega_{\mathrm{x}}$ once a value for $\mathrm{A}_{\mathrm{x}}$ has been chosen.

b) Fixing a value for $\gamma_{x}$ and determining $\gamma_{y}$ so that $M_{e}\left(\tau_{0}\right)$ has no sign changes. In this case, the equation:

$$
\frac{4 \delta-\gamma_{x}\left(I_{1}-I_{2}\right)}{I_{3}}=\frac{A_{y} \omega_{y}^{2}}{g}
$$

allows to calculate $\omega_{\mathrm{y}}$ from a fixed value of $\mathrm{A}_{\mathrm{y}}$.

To analyze the previous results, Fig 11 a) shows the variation of $A_{x}$ as a function of the frequency $\omega_{\mathrm{x}}$ taking $\omega_{\mathrm{y}}=4.89 \mathrm{rad} / \mathrm{s}$ for different amplitudes $\mathrm{A}_{\mathrm{y}}$ in accordance with Eq (72). Similarly, Fig 11 b) shows the variation of $A_{y}$ as a function of the frequency $\omega_{\mathrm{y}}$ taking $\omega_{\mathrm{x}}=3.77 \mathrm{rad} / \mathrm{s}$ for different amplitudes $\mathrm{A}_{\mathrm{x}}$ taking into account $\mathrm{Eq}$ (73). It should be noticed that points $P_{1}(3.77,0.9)$ and $P_{2}(4.89,0.8)$ are above the curves for $\mathrm{A}_{\mathrm{x}}$ and $\mathrm{A}_{\mathrm{y}}$, i.e. they are in a zone where the Melnikov function has zeros and therefore chaotic behavior may occur. However, points $\mathrm{P}_{3}(3.77,0.2)$ and $\mathrm{P}_{4}(4.89,0.2)$ are below the curves for $A_{x}$ and $A_{y}$, so the Melnikov function has no zeros and thus chaotic behavior is impossible. It is interesting to remark that the Melnikov function is almost symmetrical around zero for the values indicated at points $\mathrm{P}_{1}$ and $\mathrm{P}_{2}$, regardless the values for the angle $\varphi$ of Eq (68), so the intersection between the stable and unstable manifolds is permanent and therefore chaotic behavior appears.

Figure 11 
To verify the occurrence of chaotic oscillations in accordance with Fig 11, Eqs (9) and (19) have been simulated taking into account the harmonic disturbances at the suspension point and the control action, which is applied in an arbitrary time [17-18] [38]. The results are plotted in the phase plane $\theta(\mathrm{t})-\mathrm{d} \theta(\mathrm{t}) / \mathrm{dt}$ as shown in Fig 12. The simulation starts with the initial condition $\theta(0)=0.4 \mathrm{rad}$, with harmonic disturbances of zero initial velocity and without control. At $\mathrm{t}=40 \mathrm{~s}$, harmonic disturbances with $\omega_{\mathrm{x}}=$ $3.77 \mathrm{rad} / \mathrm{s}, \mathrm{A}_{\mathrm{x}}=0.9 \mathrm{~m}, \omega_{\mathrm{y}}=4.89 \mathrm{rad} / \mathrm{s}$ and $\mathrm{A}_{\mathrm{y}}=0.8 \mathrm{~m}$ are applied (points $\mathrm{P}_{1}$ and $\mathrm{P}_{2}$ of Fig 11). It should be noted that the angle $\theta(t)$ is reduced to its equivalent value between 0 and $2 \pi$ and that the velocity seems to oscillate chaotically.

Assuming that the strange attractor of Fig 12 is chaotic, there will always be an orbit that is very close to the pointing-up position $\theta_{\mathrm{e}}=\pi$. Consequently, when a chaotic orbit crosses a predefined capture region $\Omega$ around $\theta_{\mathrm{e}}$, it is possible to drive the motion of the pendulum around the weak focus $\theta_{\mathrm{e}}=\pi$ by applying the PID control defined by Eq (8). The capture region $\Omega$ is defined through the amplitudes $r_{a x}$ and $r_{a y}$ of the angle $\theta(\mathrm{t})$ and the angular velocity $\mathrm{d} \theta(\mathrm{t}) / \mathrm{dt}$ respectively. However, it should be pointed out that we don't know a priori the exact moment at which a chaotic orbit will be close to the set point, so the capture region must be properly chosen to avoid long waiting times before applying the control.

When the chaotic motion is consolidated, a chaotic orbit may enter a capture zone $\Omega$ defined by $\mathrm{r}_{\mathrm{ax}}=0.6 \mathrm{rad}$ and $\mathrm{r}_{\mathrm{ay}}=0.6 \mathrm{rad} / \mathrm{s}$ from the (arbitrarily chosen) instant $\mathrm{t}_{\mathrm{c}}$ $=320 \mathrm{~s}$. When the PID control law acts at the instant $\mathrm{t}_{\mathrm{ii}}=323.24 \mathrm{~s}$, the chaotic motion is destroyed but the pendulum remains with irregular oscillations caused by the harmonic disturbances at the suspension point O'. Finally, such harmonic disturbances are removed at $\mathrm{t}=370 \mathrm{~s}$, and the pendulum remains with a damped regular oscillation around the weak focus.

\section{Figure 12}

Fig 13 shows that the pendulum dynamic is chaotic for the values of the harmonic disturbances (at the suspension point) indicated at points $\mathrm{P}_{1}$ and $\mathrm{P}_{2}$ of Fig 11 . Fig 13 a) shows the sensible dependence for two initial conditions $\theta(t)$ and $\theta_{1}(t)$ that initially differ in $10^{-8}$. The solutions are completely different from $\mathrm{t} \approx 250 \mathrm{~s}$, but they become coincident once the chaotic motion has been removed. Fig 13 b) shows the 
calculation of all Lyapunov exponents based on the algorithm described in refs. [41-42], for which a Matlab software has been implemented [43].

It should be noted that the presence of a positive Lyapunov exponent is a typical indicator of chaotic behavior. Besides, the sum of the Lyapunov exponents is -1 at $\mathrm{t}_{\mathrm{c}}=320 \mathrm{~s}$, i.e. it coincides with the divergence of vector field $\operatorname{div}(f)=-\delta=-1$ (see Eq (20)) and therefore the simulation results can be regarded as correct. In Fig 13 c), the power spectral density shows the presence of a continuous non periodic spectrum, which is also a typical feature of chaotic motion [36]. On the other hand, Fig $13 \mathrm{~d}$ ) shows the required values of the forces to obtain chaotic motion according to Eqs (5) and (6). It should be remarked that these values are similar to the ones shown in Fig $3 \mathrm{c}$ ) and they are small in comparison with the high-frequency oscillations shown in Fig 4 b).

Figure 13

The system subjected to harmonic excitations at the suspension point is not autonomous, so it is convenient to transform it into an autonomous one by introducing in Eqs (9) the auxiliary variables $\mathrm{z}_{\mathrm{i}}(\mathrm{t})(\mathrm{i}=1,2,3,4,5)$ given by:

$$
\begin{gathered}
z_{1}(t)=\theta(t) ; z_{2}(t)=\dot{\theta}(t) ; z_{3}(t)=u(t) \\
\frac{d z_{4}(t)}{d t}=\omega_{x}, z_{4}(t) \bmod 2 \pi ; \frac{d z_{5}(t)}{d t}=\omega_{y}, z_{5}(t) \bmod 2 \pi \\
f_{\omega x}=\omega_{x} t ; f_{\omega y}=\omega_{y} t
\end{gathered}
$$

Figs 14 a) and b) show the auxiliary variable $z_{5}(t)$ defined in Eqs (74) as a function of the state variables $z_{1}(t)$ and $z_{2}(t)$, whose Poincaré sections plotted in Figs $14 \mathrm{c}$ ) and $14 \mathrm{~d}$ ) with $\mathrm{f}_{\omega \mathrm{y} 1}=4$ and $\mathrm{f}_{\omega \mathrm{y} 2}=2$ show a clear chaotic behavior. The previous results together with the necessary conditions deduced from the Melnikov function given by Eqs (68)(71) allow us to affirm that the strange attractor of Fig 12 is chaotic.

Figure 14

In section 4 we investigated the conditions under which the analytical results deduced from the normal forms are very close to the numerical simulations of the 
system. The same arguments can now be considered when the chaotic dynamics is removed and a weak focus appears in the pointing-up position. In accordance with the results of Fig 12, the harmonic disturbances at the suspension point $\mathrm{O}^{\prime}$ are removed at $\mathrm{t}$ $=370 \mathrm{~s}$. For $\mathrm{K}_{\mathrm{p}}=5.4444 \mathrm{~N} \cdot \mathrm{m}, \tau_{\mathrm{i}}=0.9989 \mathrm{~s}$ and $\tau_{\mathrm{d}} 10^{-3} \mathrm{~s}$, Eqs (37)-(39) allow to obtain that $b^{0}=0.1536$ and $b^{1}=-0.0768+0.2359 i$, which can be considered as sufficiently small values so that the approximations $x_{1}(t) \approx y_{1}(t)$ and $x_{0}(t) \approx y_{0}(t)$ are fulfilled and thus similar results to the ones of Fig 8 are expected.

To verify this issue, Figs $15 \mathrm{a}$ ) and b) show the values of $\mathrm{y}_{0}(\mathrm{t})$ and $\mathrm{y}_{1}(\mathrm{t})$ deduced from the inverse normalizing transformations as well as their comparison with the analytical values deduced from Eqs (52)-(53). In Figs 15 c) and d), the values of the pendulum state variables deduced from the simulation of Eqs (9) and (19) are compared with the values $z z_{i}{ }_{i}(t) i=1,2$ deduced from Eqs (25) and (27) by using the inverse normalizing transformations. Since the values are very similar, we have obtained another confirmation of the hypothesis regarding the choice of the PID controller parameters as well as of the analytical derivations and the numerical simulations of the system.

Figure 15

To investigate a possible application of the chaotic behavior and the robustness of the PID controller, Fig 16 shows a strange attractor obtained in a similar way to the one of Fig 12. It is assumed that when a chaotic orbit enters a capture zone $\Omega\left(r_{a x}=0.5\right.$ $\mathrm{rad}, \mathrm{r}_{\mathrm{ay}}=0.5 \mathrm{rad} / \mathrm{s}$ ) around $\theta_{\mathrm{e}}=\pi$, the parameters of the PID controller are changed so that the weak focus associated to the pointing-up position is destroyed and $\theta_{\mathrm{e}}=\pi$ becomes an asymptotically stable equilibrium point. It is interesting to remark that the attractor can be more or less dense in a neighborhood of $\theta_{\mathrm{e}}=\pi$ depending on the values for $A_{x}, \omega_{x}, A_{y}$ and $\omega_{y}$, so the capture region should be defined accordingly. On the other hand, it should be recalled that the system is of third-order once the harmonic disturbances are eliminated $\left(\ddot{x}_{0}=0, \ddot{y}_{0}=0\right)$, so we can define a settling time $t_{\mathrm{s}}$ and a new damping coefficient $0<\delta_{\mathrm{n}}<1$ to obtain a pair of dominant roots in the characteristic equation for the Jacobian of Eqs (9), i.e. [42-43]: 


$$
\begin{aligned}
& t_{s}=\frac{4.6}{\delta_{n} \omega_{n}} ; \lambda_{1,2}=-\delta_{n} \omega_{n} \pm i \omega_{n} \sqrt{1-\delta_{n}^{2}} ; \lambda_{3}=-c ; c=-n_{1} \delta_{n} \omega_{n} \quad\left(n_{1} \geq 5\right) \\
& \left(\lambda-r_{1}\right)\left(\lambda-r_{2}\right)\left(\lambda-r_{3}\right)=\lambda^{3}+\left(c+2 \delta_{n} \omega_{n}\right) \lambda^{2}+\left(\omega_{n}^{2}+2 \delta_{n} \omega_{n} c\right) \lambda+\omega_{n}^{2} c
\end{aligned}
$$

Once $\mathrm{t}_{\mathrm{s}}, \delta_{\mathrm{n}}$ and $\mathrm{n}_{1}$ have been selected, the roots $\lambda_{1,2}$ are dominant and the new PID parameters are calculated by identifying the coefficients of the characteristic equation (Eq (21)) with the corresponding coefficients of $E q(75)$. Taking $A_{x}=0.9 \mathrm{~m}$, $\omega_{\mathrm{x}}=3.77 \mathrm{rad} / \mathrm{s}, \mathrm{A}_{\mathrm{y}}=0.8 \mathrm{~m}, \omega_{\mathrm{y}}=4.89 \mathrm{rad} / \mathrm{s}$ and $\mathrm{f}=1.5$, the pendulum reaches chaotic behavior. At $t_{c}=320 \mathrm{~s}$, the PID controller is applied assuming $K_{p}=9.8$ N.m, $\tau_{i}=1.9616$ $\mathrm{s}$ and $\tau_{\mathrm{d}}=10^{-3} \mathrm{~s}$, and the chaotic behavior is destroyed when a chaotic trajectory intersects the capture zone $\Omega$ (point $P_{1}$ of Fig 16) at the instant $t=322.69 s>t_{c}=320 s$. Nevertheless, the pendulum remains with irregular oscillations around the set point because of the harmonic disturbances. Such harmonic disturbances (at the suspension point $\mathrm{O}^{\prime}$ ) are removed at $\mathrm{t}=350 \mathrm{~s}$, when the $\mathrm{PID}$ parameters are changed to $\mathrm{K}_{\mathrm{p}}=$ 19.1183 N.m, $\tau_{\mathrm{i}}=1.6850 \mathrm{~s}, \tau_{\mathrm{d}}=0.2668 \mathrm{~s}\left(\mathrm{t}_{\mathrm{s}}=2 \mathrm{~s}, \delta_{\mathrm{n}}=0.95, \mathrm{n}_{1}=5\right)$ in accordance with Eqs (75).

To verify that the designed control law is robust against measurement uncertainties, a uniform random distribution given by $\mathrm{Eq}$ (15) has been added to the angle and angular velocity for $t>350 \mathrm{~s}$ (point $\mathrm{P}_{2}$ of Fig 16) taking $\mathrm{f}_{\mathrm{na}}=0.5$. The simulation results for the pendulum stabilization are shown in Fig 16. It should be remarked that the pendulum is driven to the set point throughout a disturbance orbit without losing its controllability and remaining in the set point with small oscillations. Finally, it is interesting to note that the considered random disturbance is much larger than the expectable one in measurement instruments, which again corroborates the robustness of the control law.

Figure 16 


\section{Conclusions}

The problem of stabilizing a simple pendulum in the pointing-up position under a control torque as well as vertical and horizontal harmonic disturbances at the suspension point has been researched by using the normal form theory and chaotic motion. Due to the harmonic disturbances at the suspension point, the chaotic motion of the pendulum in conjunction with the control torque generated by a PID controller can be used to swing up and control the pendulum in the pointing-up position, even in presence of noise.

It is known that a pendulum can be stabilized at different angles by applying vertical and horizontal excitations of high-frequency at the suspension point. However, such procedure has the inconvenience that large accelerations and forces may be necessary to stabilize the pendulum in the pointing-up position, which in addition depends on the initial conditions and can be destroyed if random disturbances are present in the system. This paper has demonstrated that the previous problems can be overcome with a control torque applied at the suspension point by means of a PID controller.

A dynamical system with a stable weak focus associated to two pure imaginary eigenvalues leads to smooth oscillations around the weak focus. To generate this motion, this paper has investigated the presence of a weak focus as a function of the PID controller parameters by means of the Routh criterion. Assuming that there are no harmonic disturbances at the pendulum suspension point, the normal form theory has been used to deduce the stability conditions for the weak focus as well as to deduce the normal form of the system. It has been demonstrated that the stability conditions are fulfilled as long as the parameters of the PID controller are chosen according to the Routh criterion.

From the results obtained from the direct and inverse normalizing transformations, a procedure to choose the PID parameters has been deduced and applied in the numerical simulations. A complete agreement between the numerical results and the analytical predictions has been obtained. It has also been shown that there is a wide range for the PID parameter values that provides a stable pointing-up 
equilibrium position, even in presence of harmonic disturbances at the pendulum suspension point. This property provides a great flexibility to obtain different smooth motions around the pointing-up position.

The possibility of chaotic behavior for the pendulum has been studied on the basis of the Melnikov's function, which has been calculated analytically from the heteroclinic orbit of the unperturbed system. We have deduced necessary conditions for chaotic behavior with vertical and horizontal harmonic disturbances of moderate frequencies, which require moderate forces at the suspension point. Since currently there is not a definitive condition to know whether an irregular oscillating motion is chaotic or not, the appearance of strange attractors has been researched in terms of sensitive dependence, Lyapunov exponents, power spectral density and Poincaré sections to predict chaotic behavior. Once again, the simulation results give clear indicators of chaotic dynamics.

The chaotic behavior has been used taking into account that the homoclinic tangle associated to a strange attractor implies that a chaotic orbit will be close to the pointing-up position. For such orbit, assuming that the harmonic disturbances of the suspension point are removed, it is shown that the PID parameter values can be changed to generate a control torque that drives the pendulum to the prescribed set point, even in presence of random disturbances. This procedure offers the additional advantage of driving the pendulum to the pointing-up position by using small control torques.

As a concluding remark, it should be pointed out that the techniques shown in this paper can be applied to a wide variety of mechanical systems. The use of the normal form theory, the chaotic behavior and the random noise have been presented from a unified viewpoint which offers many opportunities for new investigations with more elaborated models. For instance, the elastic properties of the pendulum together with the normal form theory and the Melnikov method could be used to analyze new kind of system motions in the case of disturbances of high frequency. 


\section{References}

[1] H. C. Corben, P. Stehle, Classical Dynamics, Dover Publications, New York, 1977.

[2] J. P. Den Hartog, Mechanical Vibrations, Dover Publications, New York, 1984.

[3] W. T. Grandy, Matthias Schöck, Simulations of nonlinear pivot-driven pendulum, Am. J. Phys. 65 (1997) 376-381.

[4] Smith H J T, Blackburn J A, Experimental study of an inverted pendulum Am. J.Phys. 60 (10) (1992) $909-911$.

[5] By M. J. Clifford, S. R. Bishop, Inverted oscillations of a driven pendulum, Proc. R. Soc. Lond. A 454 (1998) 2811-2817.

[6] Ya-Xuan Zhang, Zhong-Jie Han, Gen-Qi Xu, Expansion of solution of an inverted pendulum system with time delay, Applied Math Computation 217 (2011) 67466489.

[7] K. Furuta, M. Yamasita, Swing up control of inverted pendulum. Proc. IECON, 91 (1991) 2193-2198.

[8] K. Furuta, M. Yamasita, S. Kobayashi, Swing-up control of inverted pendulum using pseudo-state feedback. J. Sys. Control Eng. 206 (1992) 263-269.

[9] M.G. Henders, A.C. Sondack, Dynamics and stability state-space of a controlled inverted pendulum. Int. J. Non Lin Mech. 31 (1996) 215-227.

[10] R. Lozano, I. Fantoni, and D. Block, Stabilization of the inverted pendulum around its homoclinic orbit. Systems \& Control Letters, 40 (2000)197-204.

[11] K.J. Astrom, K. Furuta, Swing-up a pendulum by energy control. Automatica, 36 (2000) 287-295.

[12] A. Shiriaev, A. Pogromsky, H. Ludvigsen, and O. Egeland, On global properties of passivity-based control of an inverted pendulum. Int. J. Robust Nonlinear Control 10 (2000).283-300.

[13] D. Angeli, Almost global stabilization of the inverted pendulum via continuous state feedback. Automatica, 37 (2001)1103-1108.

[14] M. Alonso, E. E. Paolini, and J. L. Moiola. Controlling an inverted pendulum with bounded controls. In L. Gruene and F. Colonius, editors, Dynamics, Bifurcations, and Control, volume 273 of Lecture Notes in Control and Information Sciences, pages 3-16. Springer-Verlag, Berlin, (2002).

[15] H. Kajiwara, P. Apkarian, and P. Gahinet. Lpv techniques for control of an inverted pendulum. IEEE Control Systems, (1999) 44-54.

[16] Sang-Yoon Kim, Bambi Hu, Bifurcations and transitions to chaos in an inverted pendulum, Phys. Rev. E, 58 (3) (1998) 3028-3035.

[17] L. Dai, M.C. Singh, Periodic, quasiperiodic and chaotic behavior of a driven Froude pendulum, Int. J. NonLin. Mech. 6 (1998) 947-965.

[18] Robert De Serio, Chaotic pendulum: The complete attractor, Am. J. Phys. 71 (2002) 250-257.

[19] A.Y.T. Lenng, J.L. Kuang, On the chaotic dynamics of a spherical pendulum with a harmonically vibrating suspension. Nonlinear Dynam. 43 (2006) 213-238.

[20] T.S Amer, M.A. Beck, Chaotic responses of a harmonically excited pendulum moving in circular path. Nonlinear Ana-Real, 10 (2009) 3196-3202.

[21] J. J. Thomsen, Some general effects of strong high-frequency excitation: Stiffening, biasing and smoothening, J. S. V. 253 (4) (2002) 807-831.

[22] J.L. Trueba, J.P. Baltanás, M.A.F. Sanjuán, A generalized perturbed pendulum. Chaos Soliton Fra. 15 (2003) 911-924.

[23] Jon Juel Thomsen, Vibrations and Stability: Advanced Theory, Analysis and Tools, second ed., Springer, Berlin, Heidelberg, 2003. 
[24] Jon Juel Thomsen, Slow high-frequency effects in mechanics: problems, solutions, potentials, Int. J. Bifurcation Chaos 15 (2005) 2799-2818.

[25] Alexander Fidlin, Nonlinear Oscillations in Mechanical Engineering, Springer, New York, 2006.

[26] Alexander Fidlin, Jon Juel Thomsen, Non-trivial effects of high-frequency excitation for strongly damped mechanical systems, Int. J. Non-Linear Mechanics 43 (2008) 569-578.

[27] Alexander D. Bruno, Local Methods in Nonlinear Differential Equations, SpringerVerlag, Berlin Heidelberg, 1989.

[28] V. M. Starzhinskii, Applied Methods in the Theory of Nonlinear Oscillations, Mir Publishers, Moscow, 1980.

[29] A. D. Bruno, V. F. Edneral, Normal Forms and Integrability of ODES Systems, Programming and Computer Software, 32 (2006) 139-144.

[30] A. D. Bruno, Local integrability of the Euler-Poisson Equations, Doklady Mathematics 74 (2006) 412-516.

[31] A. D. Bruno, Analysis of the Euler-Poisson equations by methods of power geometry and normal form, J. Applied Mathematics and Mechanics, 71 (2007) 168199.

[32] Manuel Perez-Molina, Manuel F. Perez-Polo, Fold-Hopf bifurcation, steady state, self-oscillating and chaotic behavior in an electromechanical transducer with nonlinear control, Commun Nonlinear Sci Numer Simulat 17 (2012) 5172-5188.

[33] J. Guckenheimer, P. Holmes, Nonlinear Oscillations, Dynamical Systems and Bifurcations of Vector Fields, Springer-Verlag, New York, 1983.

[34] S. Wiggins, Introduction to Applied Nonlinear Dynamical Systems and Chaos, second ed., Springer, New York, 2000.

[35] Lichtenberg, A.J., Lieberman, M.A.: Regular and chaotic dynamics, Springer, New York (1992)

[36] L Perko, Differential Equations and Dynamical Systems, third ed., Springer, New York, 2001.

[37] Ruihong Li, Wei Xu, Shuang Li, Chaos controlling of extended nonlinear Liénard system based on the Melnikov theory, Applied Math Computation, 176 (2006) 405-414

[38] M. F Perez-Polo, M. Perez-Molina., J. Gil Chica, Swing-up and positioning control of an inverted wheeled cart pendulum system with chaotic balancing motions. International Journal of Non-Linear Mechanics. 47, 655-665 (2012).

[39] K. Ogata, Modern Control Engineering. Prentice-Hall, New York, 2000.

[40] A.H. Nayfeh, Perturbation Methods,Wiley Interscience, New York, 1973.

[41] G. Benettin, L. Galgani, A. Giorgilly, J.M. Strelcyn, Lyapunov characteristic exponents for smooth dynamical systems and for Hamiltonian systems: a method for computing all of then, Part I: Theory, Meccanica 15(1980) 9-20.

[42] G. Benettin, L. Galgani, A. Giorgilly, J.M. Strelcyn, Lyapunov characteristic exponents for smooth dynamical systems and for Hamiltonian systems: a method for computing all of then, Part II: Numerical applications, Meccanica 15(1980) 2130.

[43] M.F.P. Polo, P. Albertos, Nonisothermal Stirred- Tank Reactor with Irreversible Exothermic Reaction A $\rightarrow$ B: Nonlinear Phenomena in: H. O. Méndez-Acosta, R. Femat, V. Gónzalez -Álvarez (Eds), Lecture Notes in Control and Information Sciences, no 361, Springer-Verlag, Berlin, Heilderberg, 2007, pp. 276-279. 


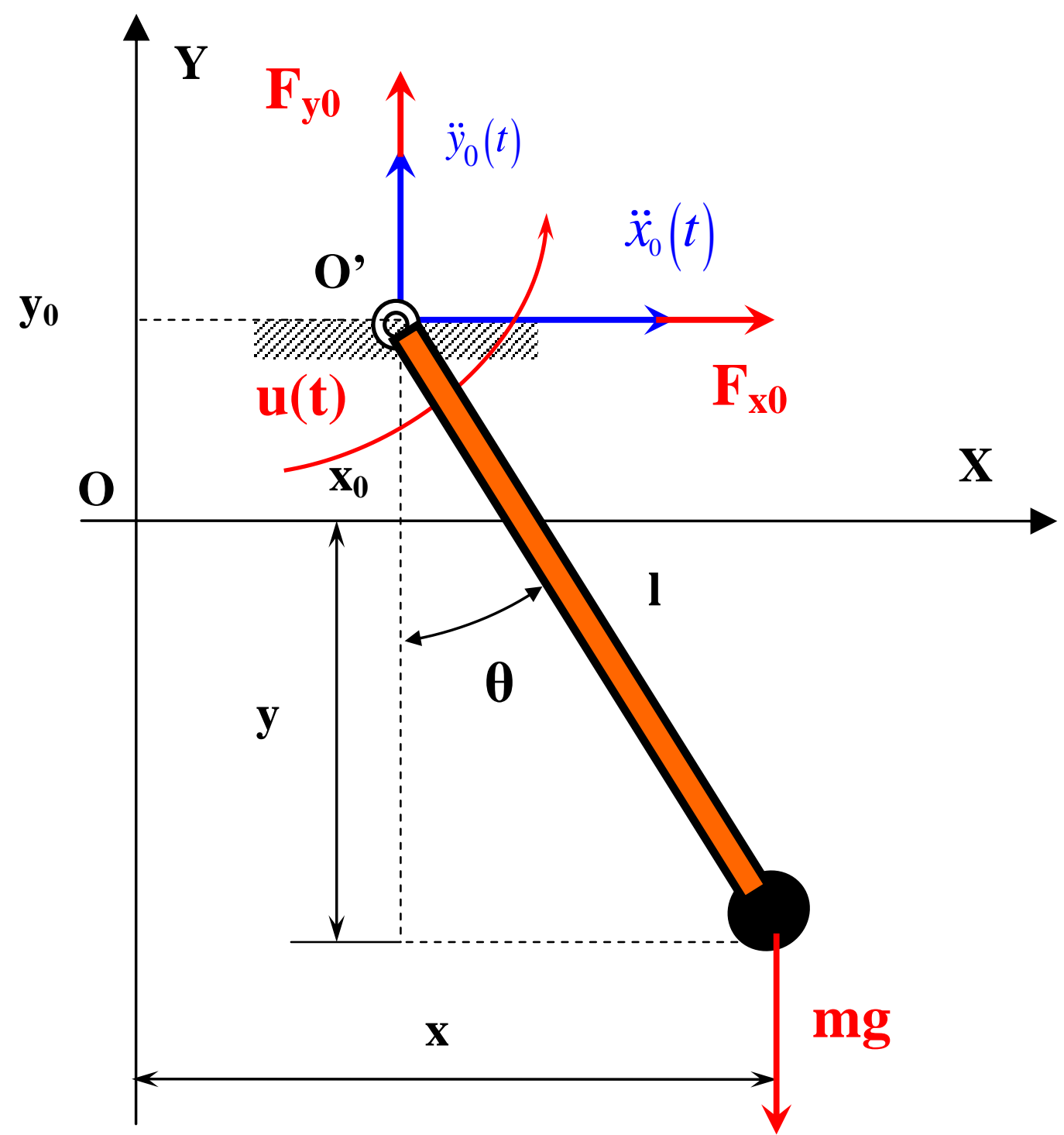

Figure 1 


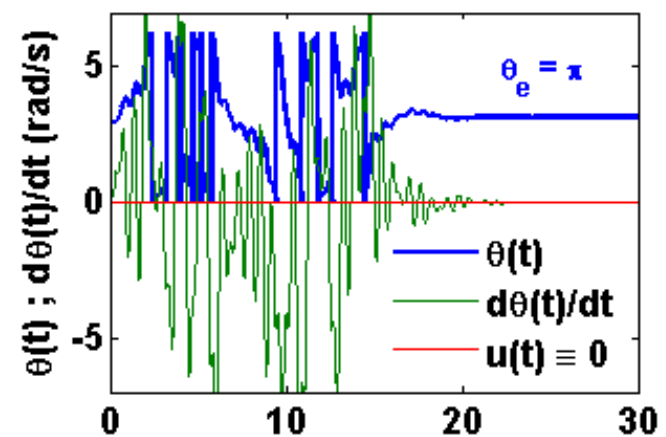

a) Time $t$ (s)

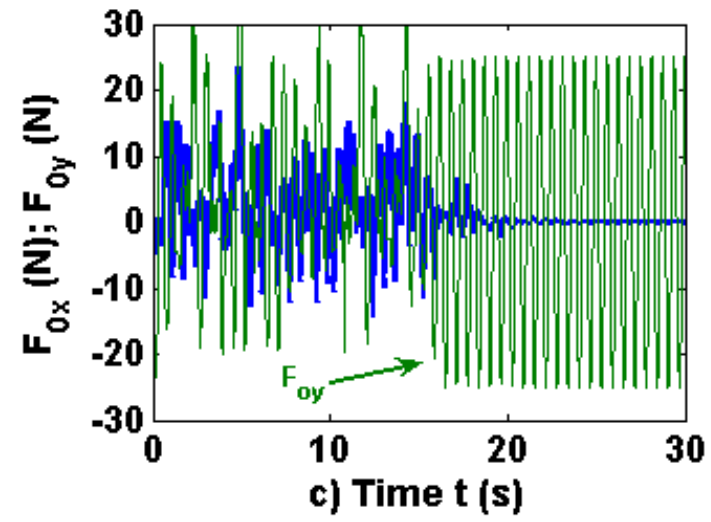

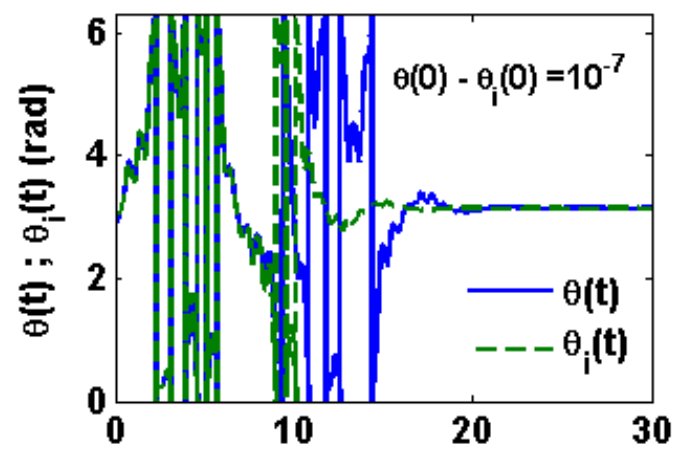

b) Time t (s)

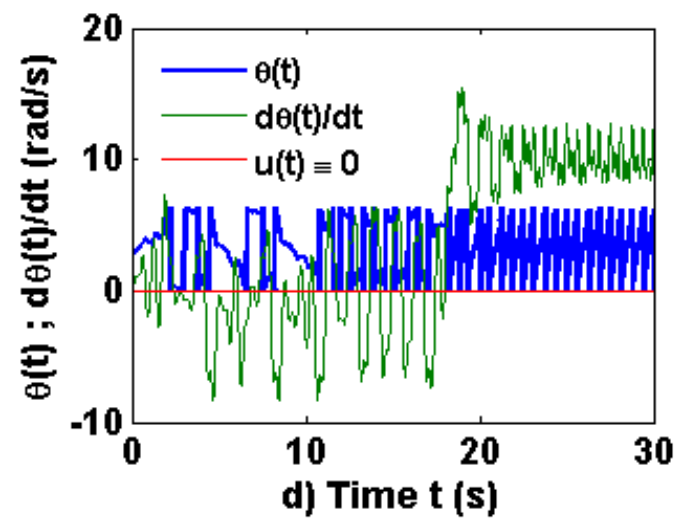

Figure 2 

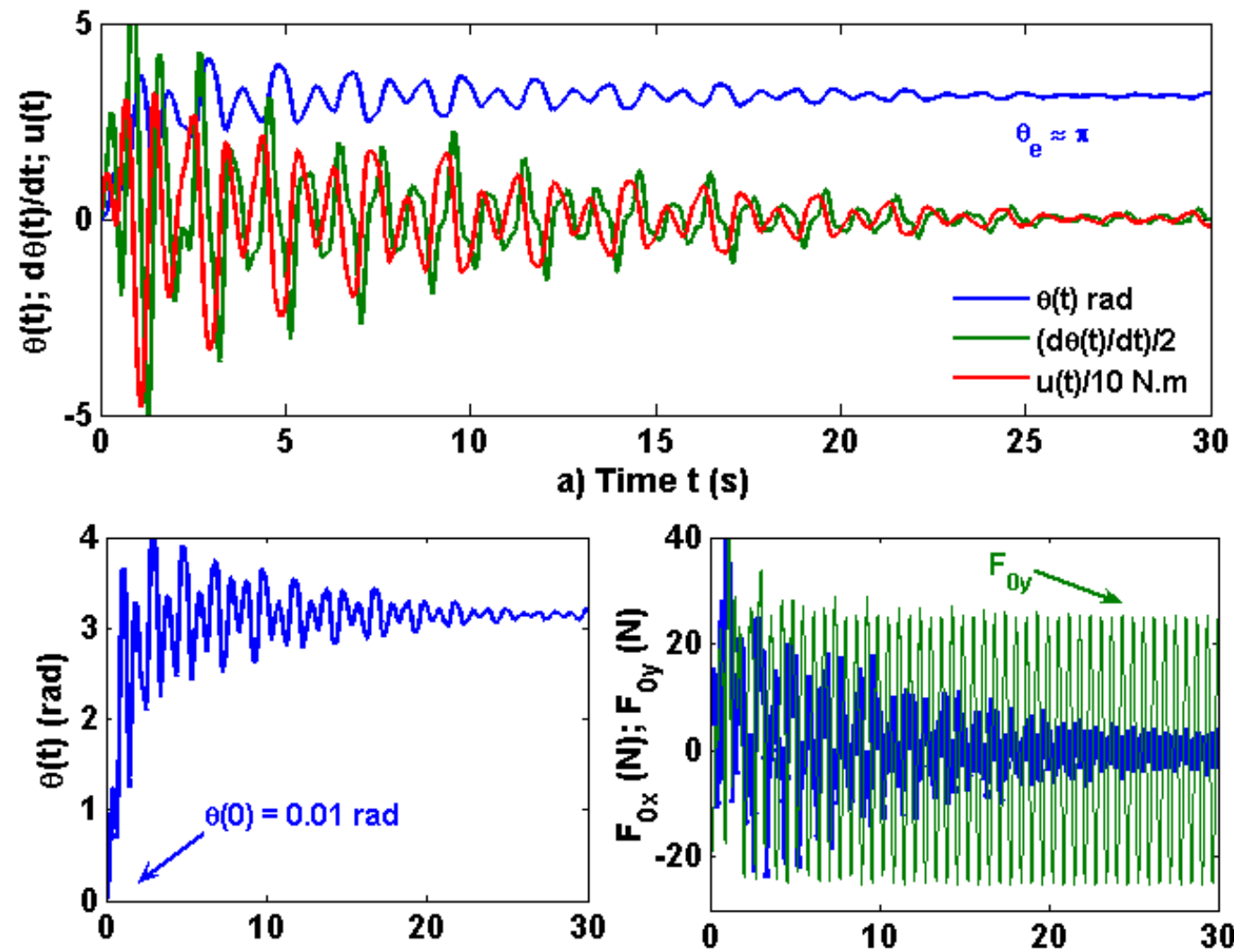

b) Time $t$ (s)

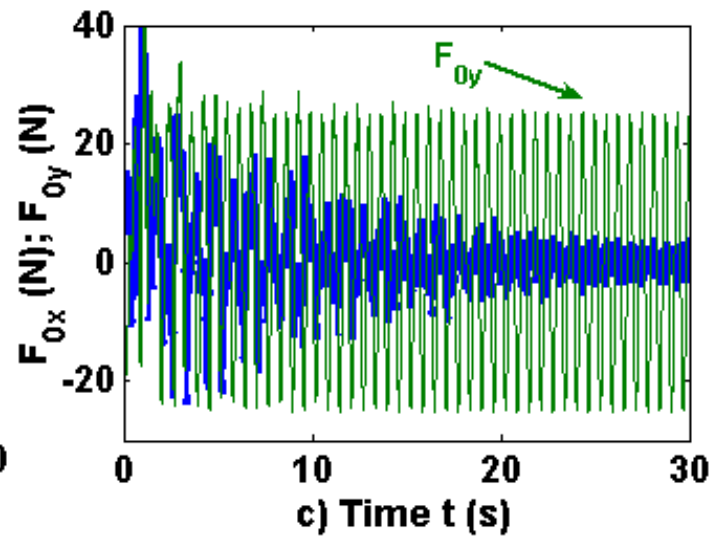

Figure 3 


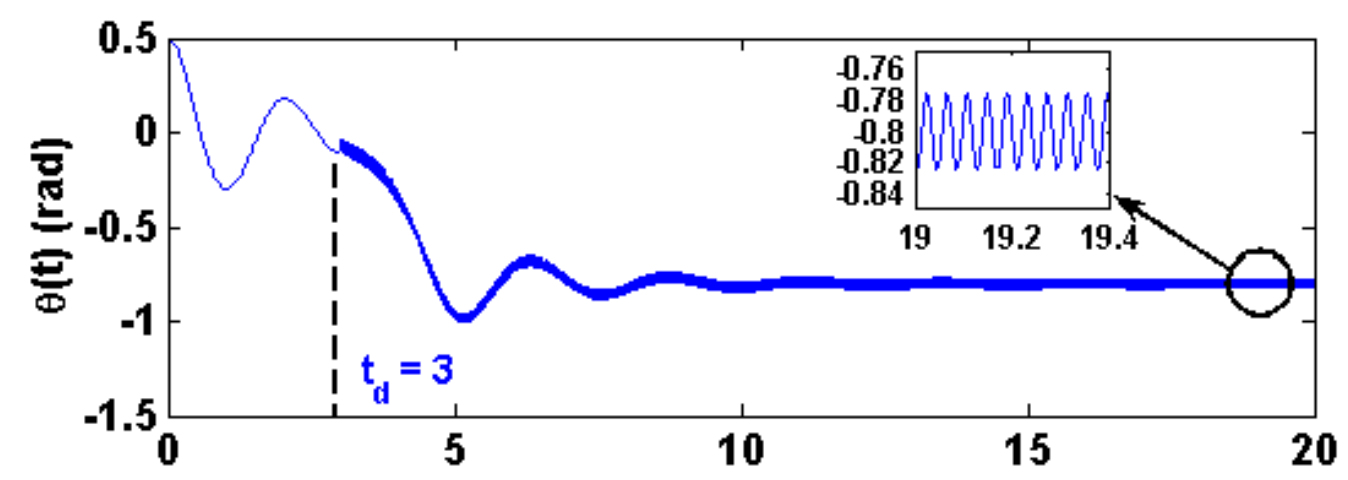

a) Time $t$ (s)

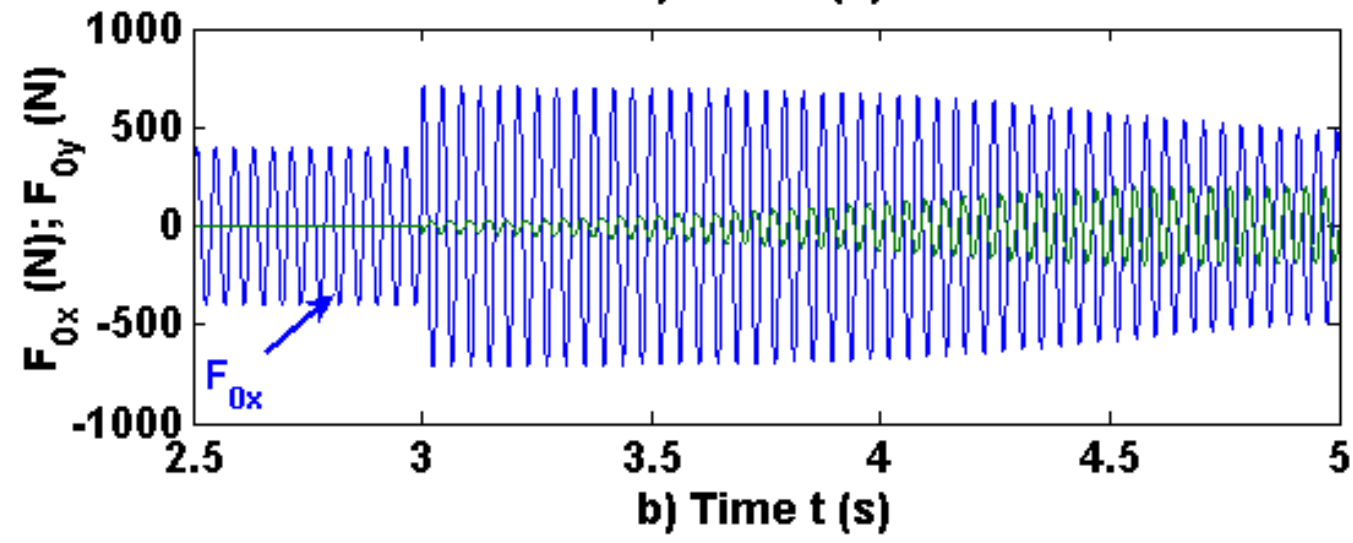

Figure 4 


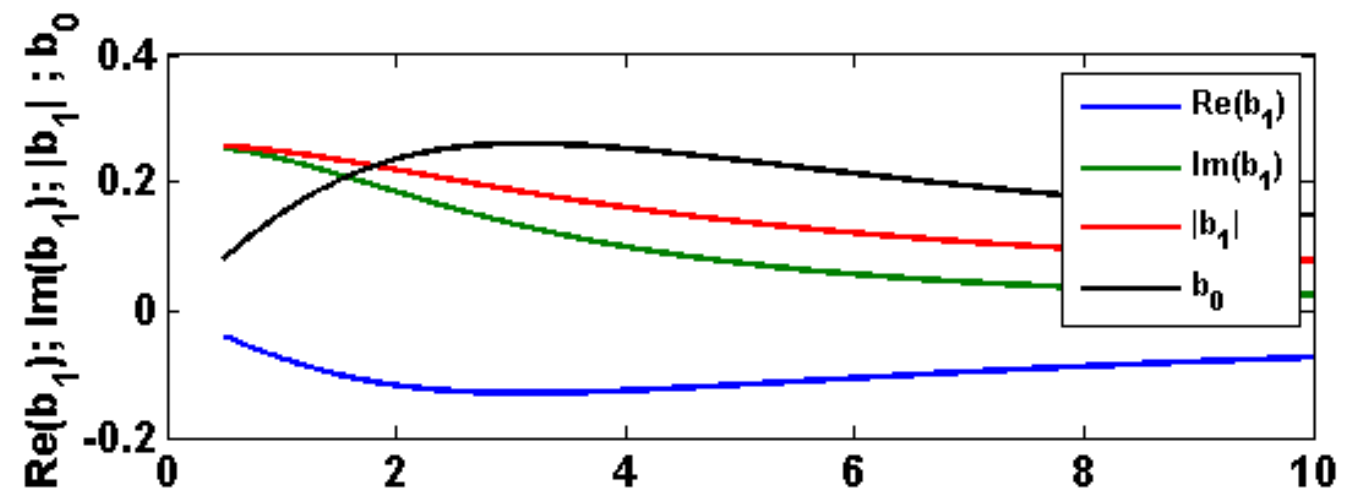

a) $\delta=b /\left(m . I^{2}\right)$

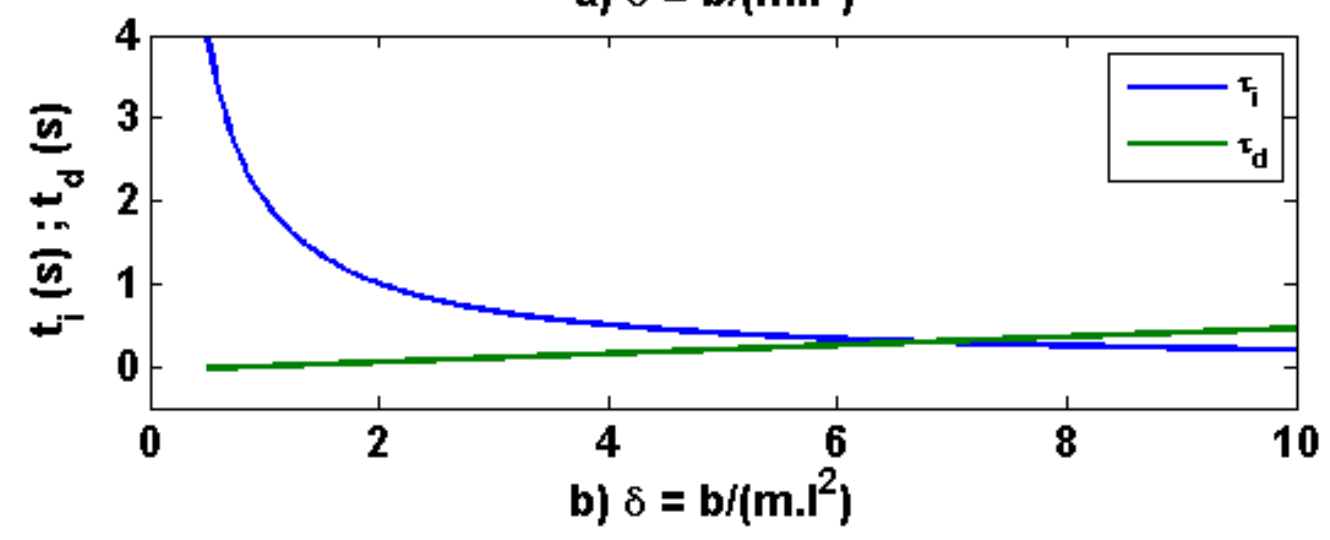

Figure 5 

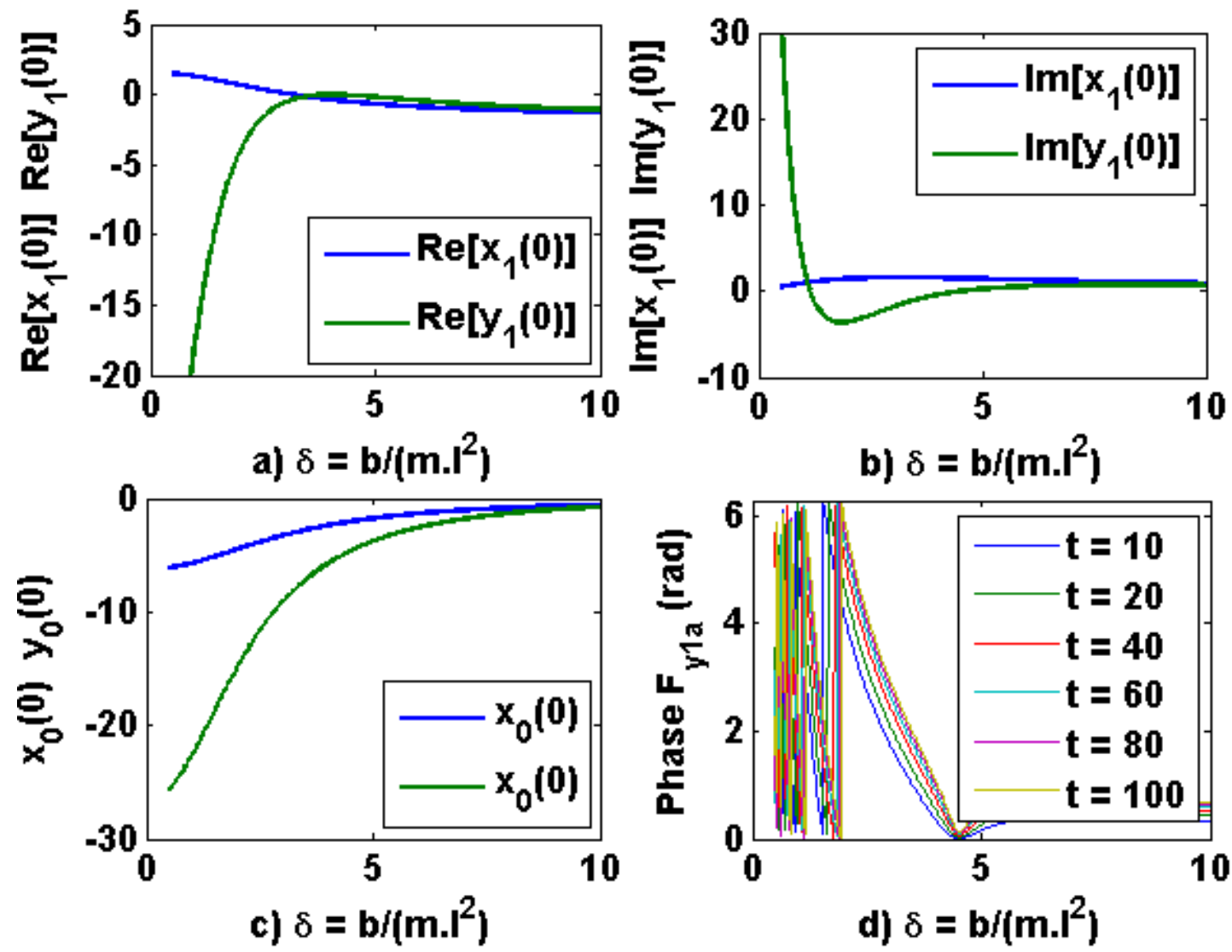

Figure 6 

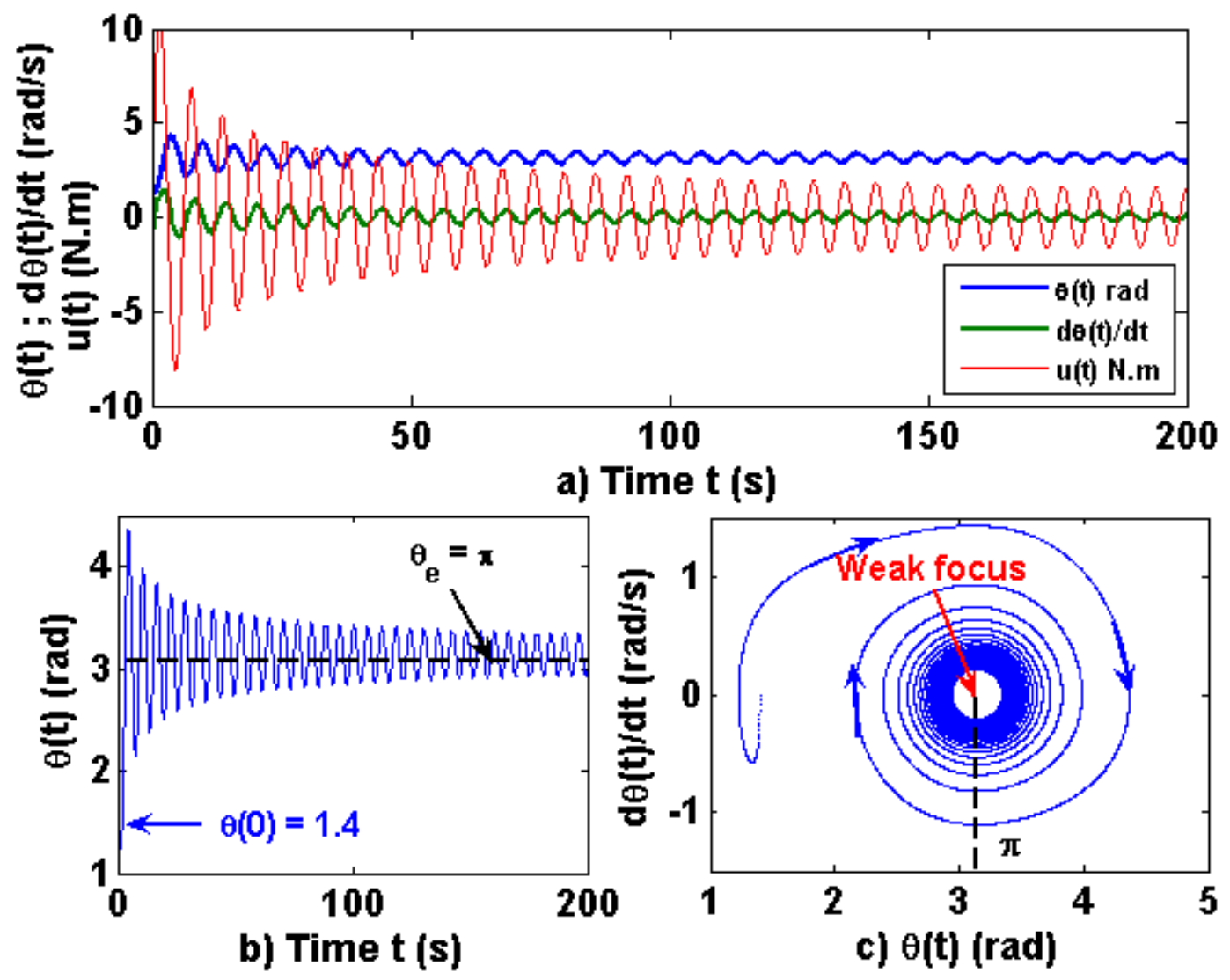

Figure 7 


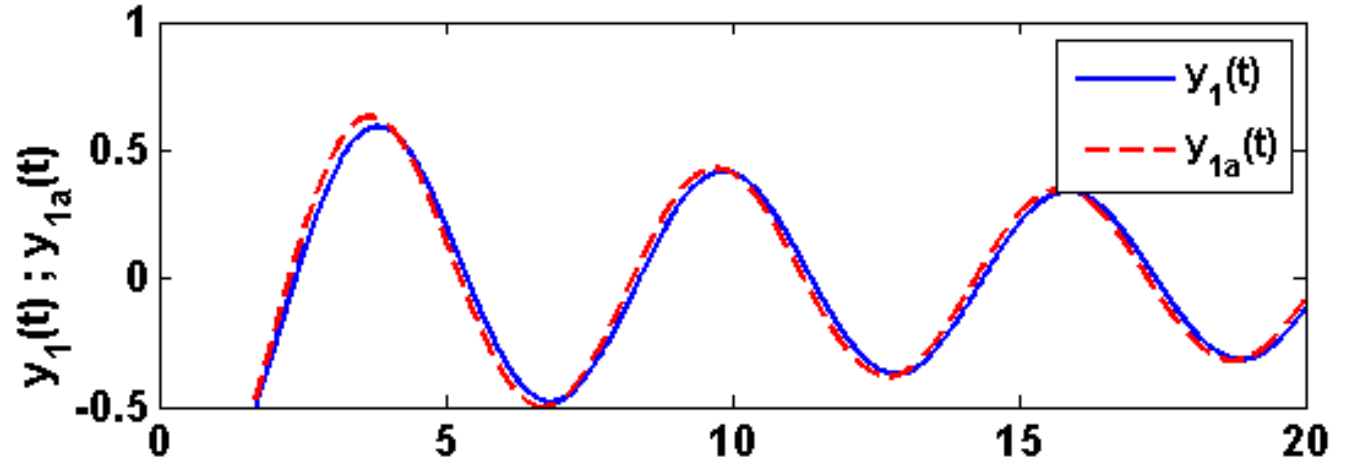

a) Time $t$ (s)

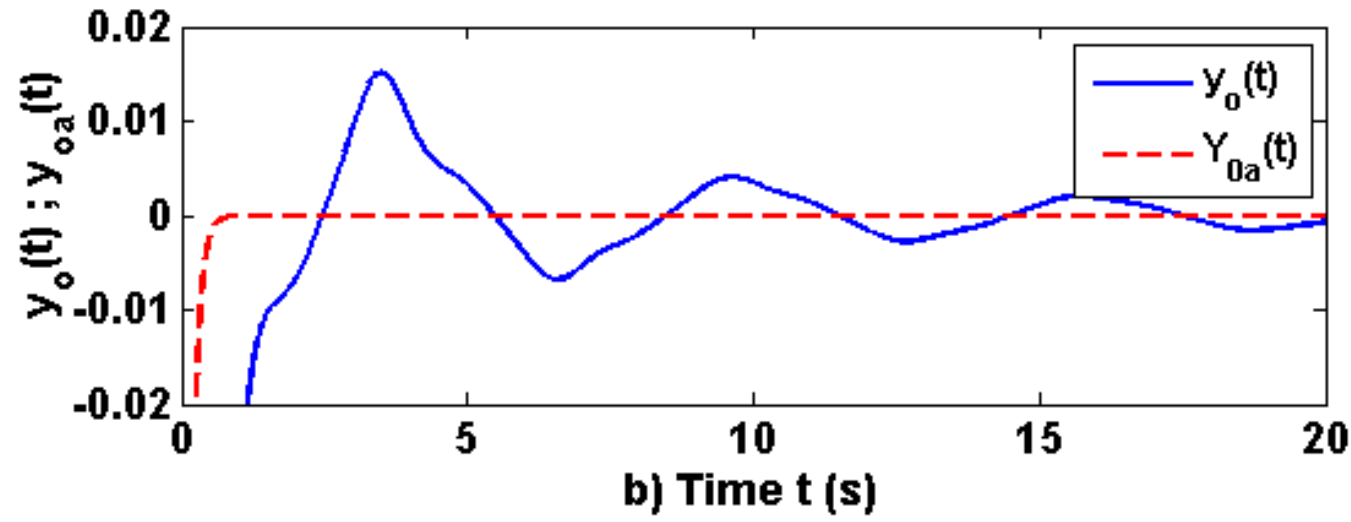

Figure 8 


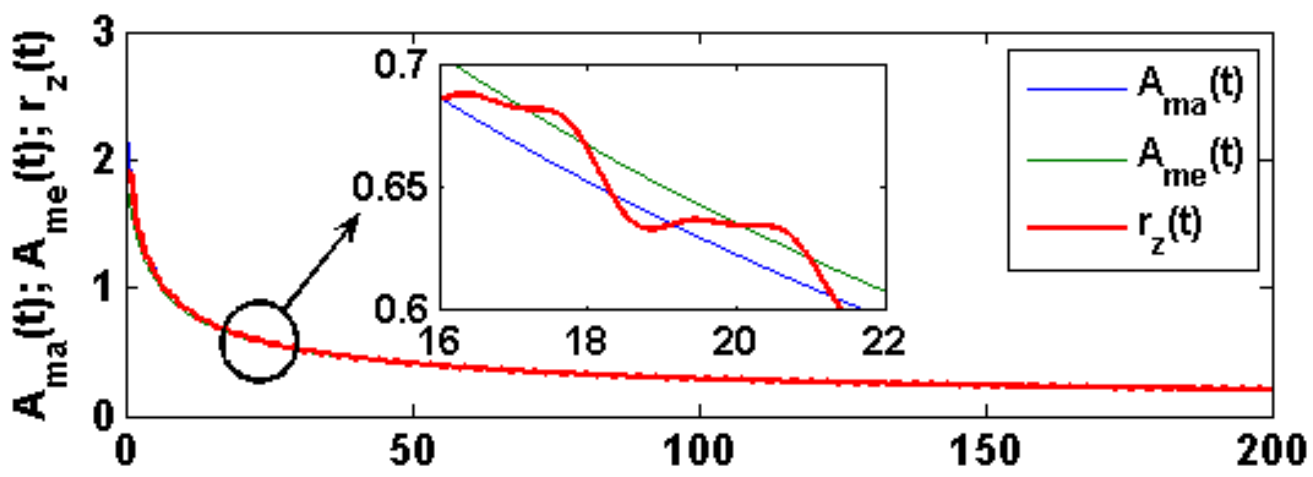

a) Time $t$ (s)

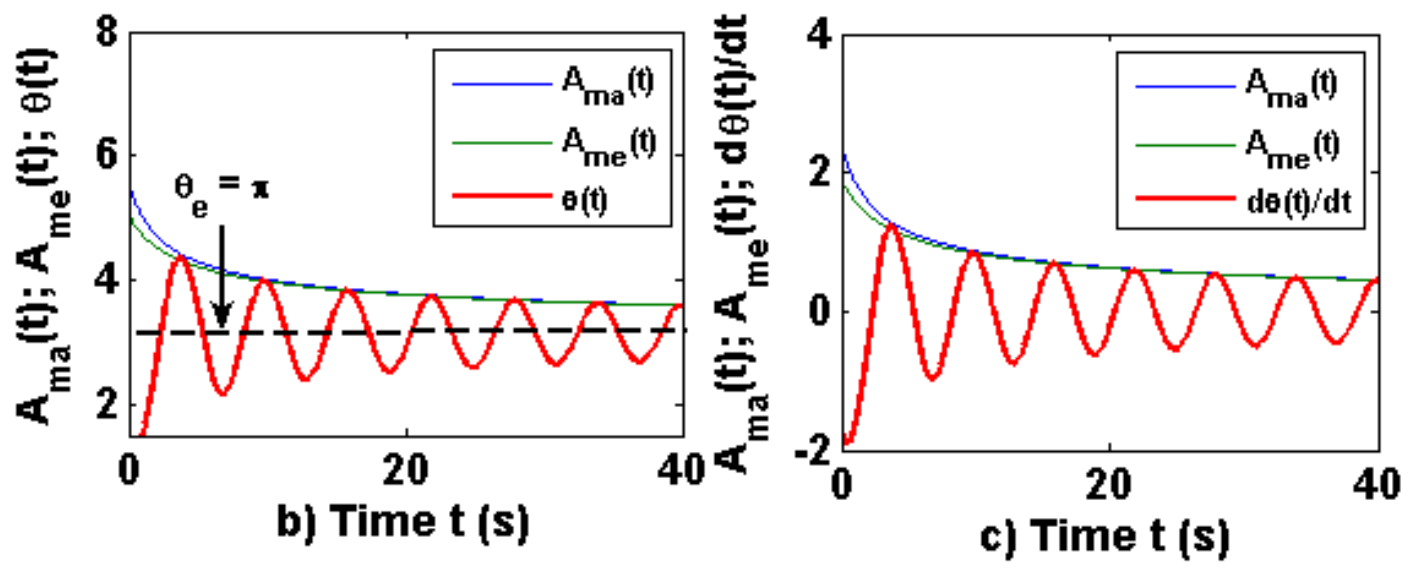

Figure 9 

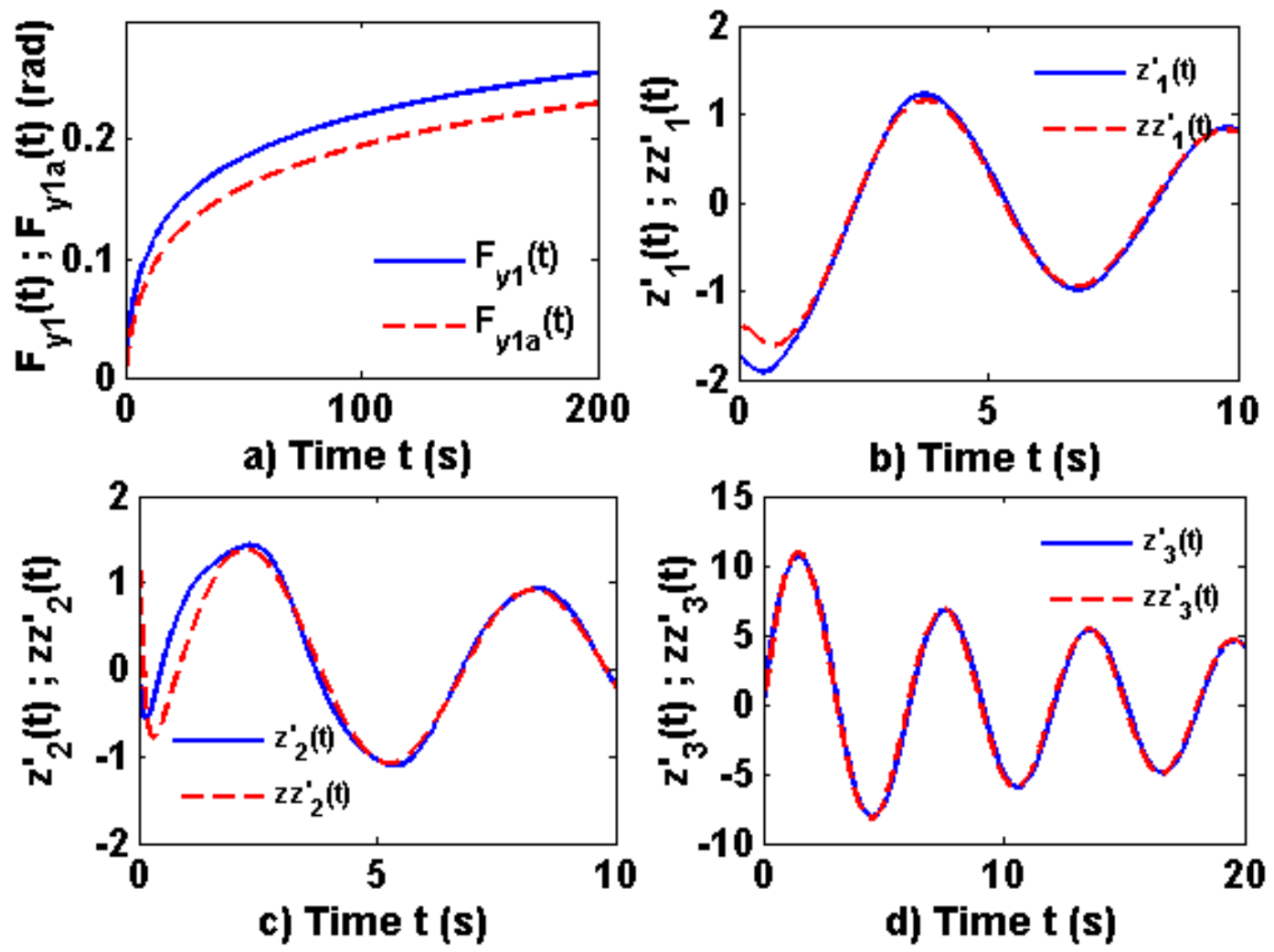

Figure 10 

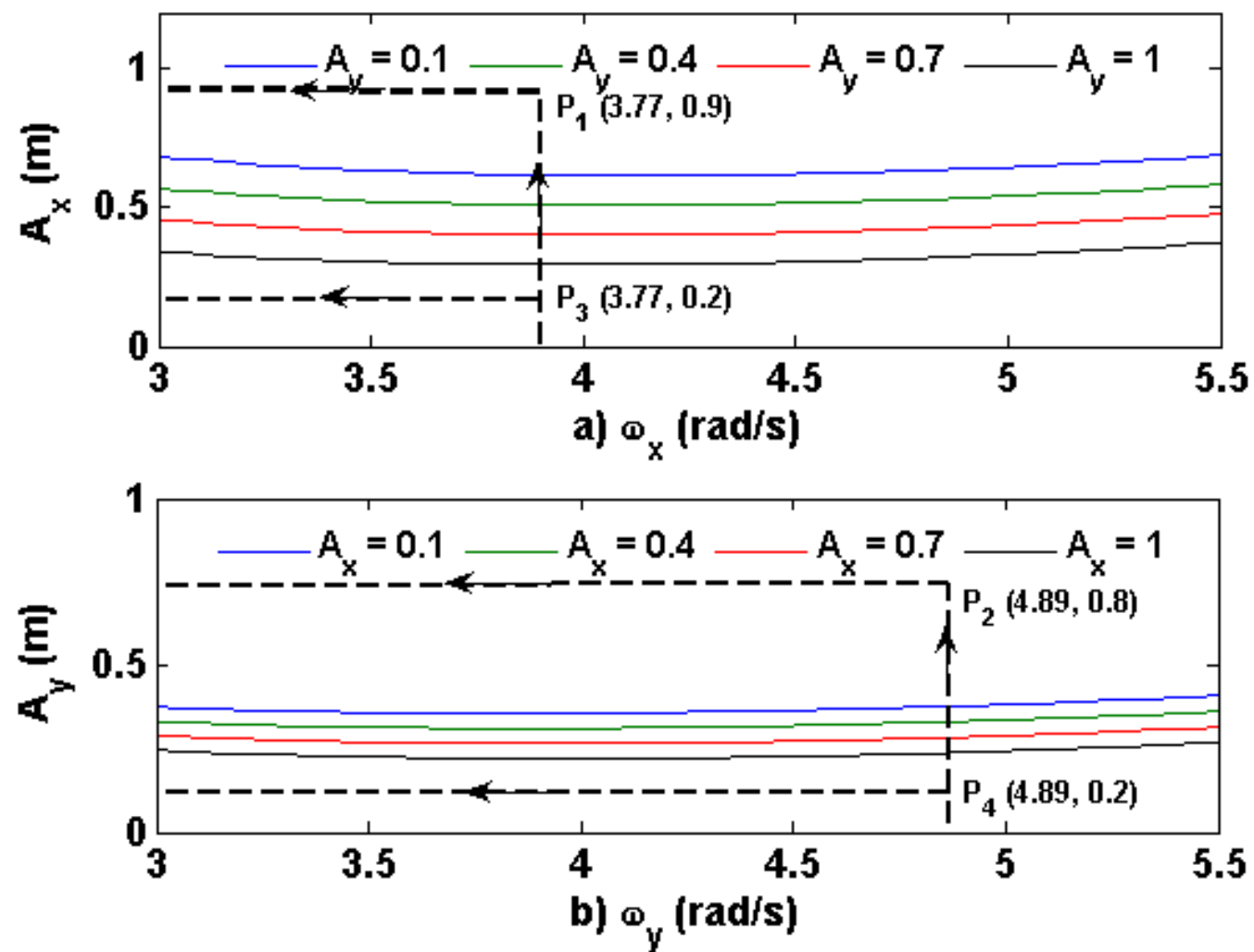

Figure 11 


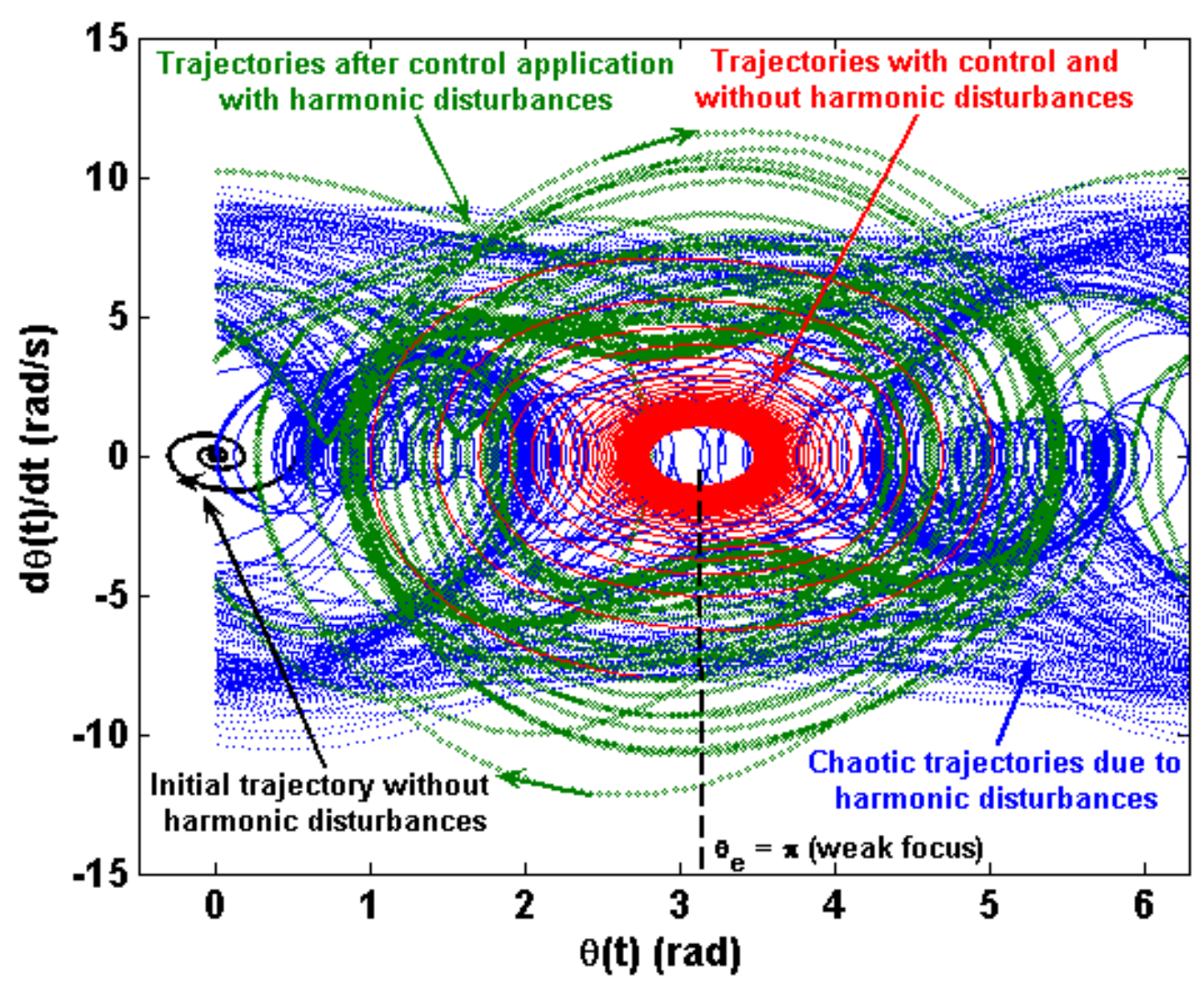

Figure 12 

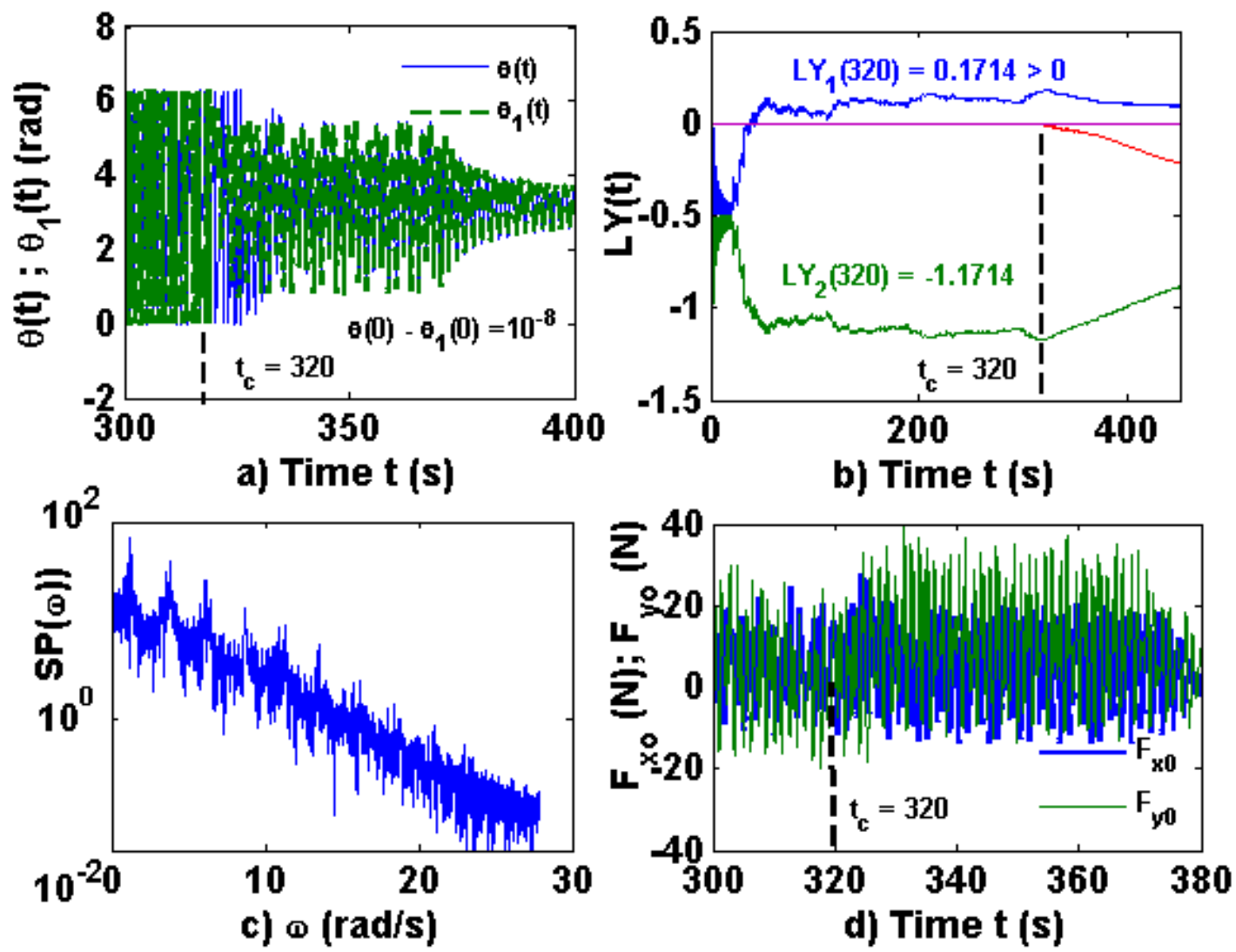

Figure 13 

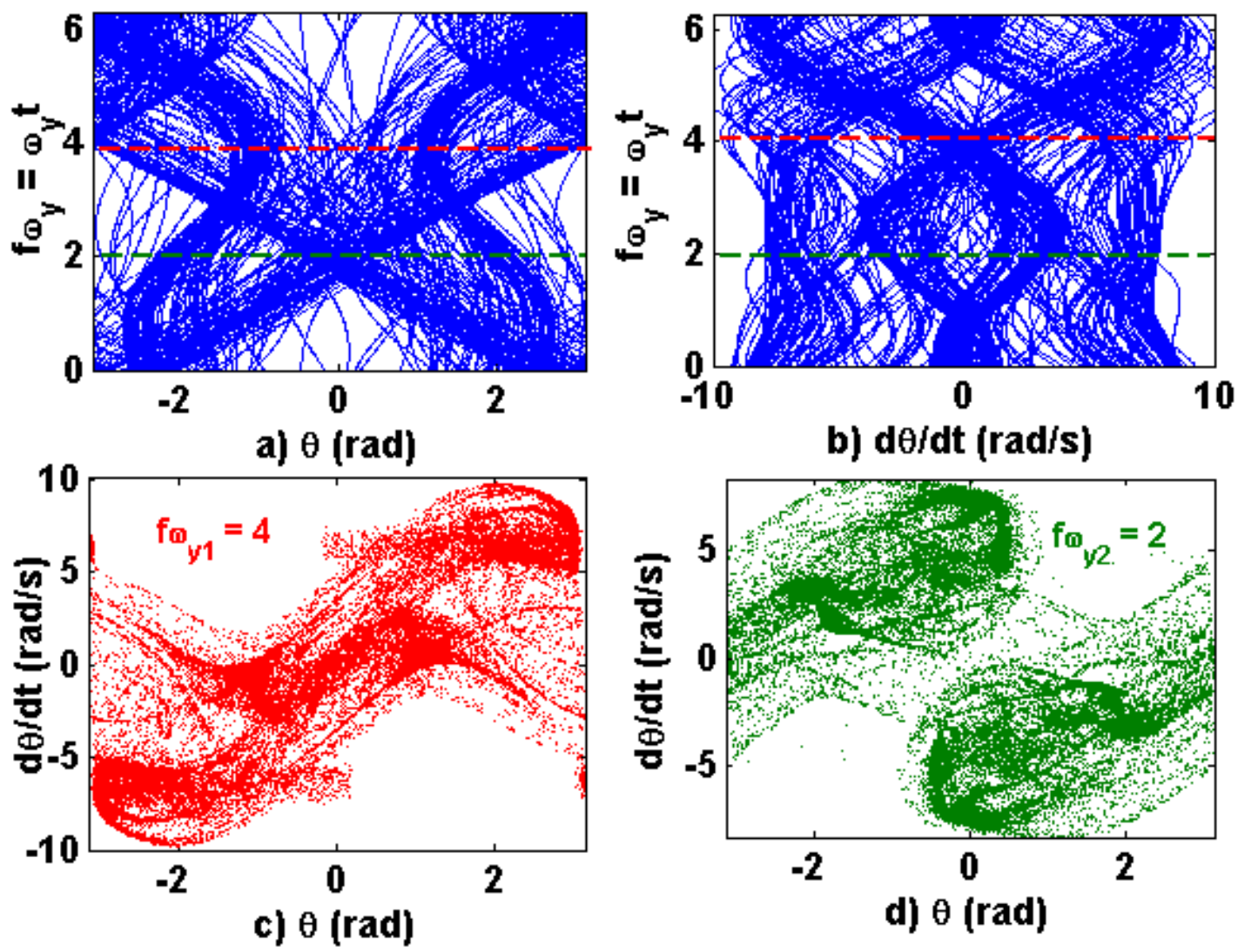

b) d $\theta / \mathrm{dt}(\mathrm{rad} / \mathrm{s})$

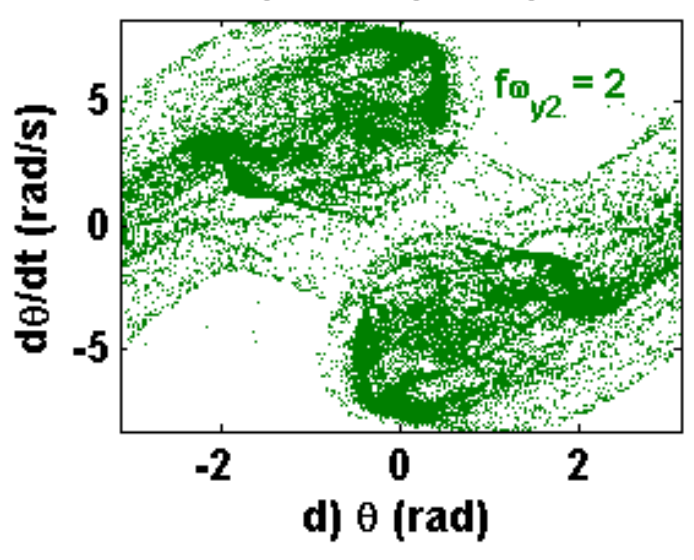

Figure 14 

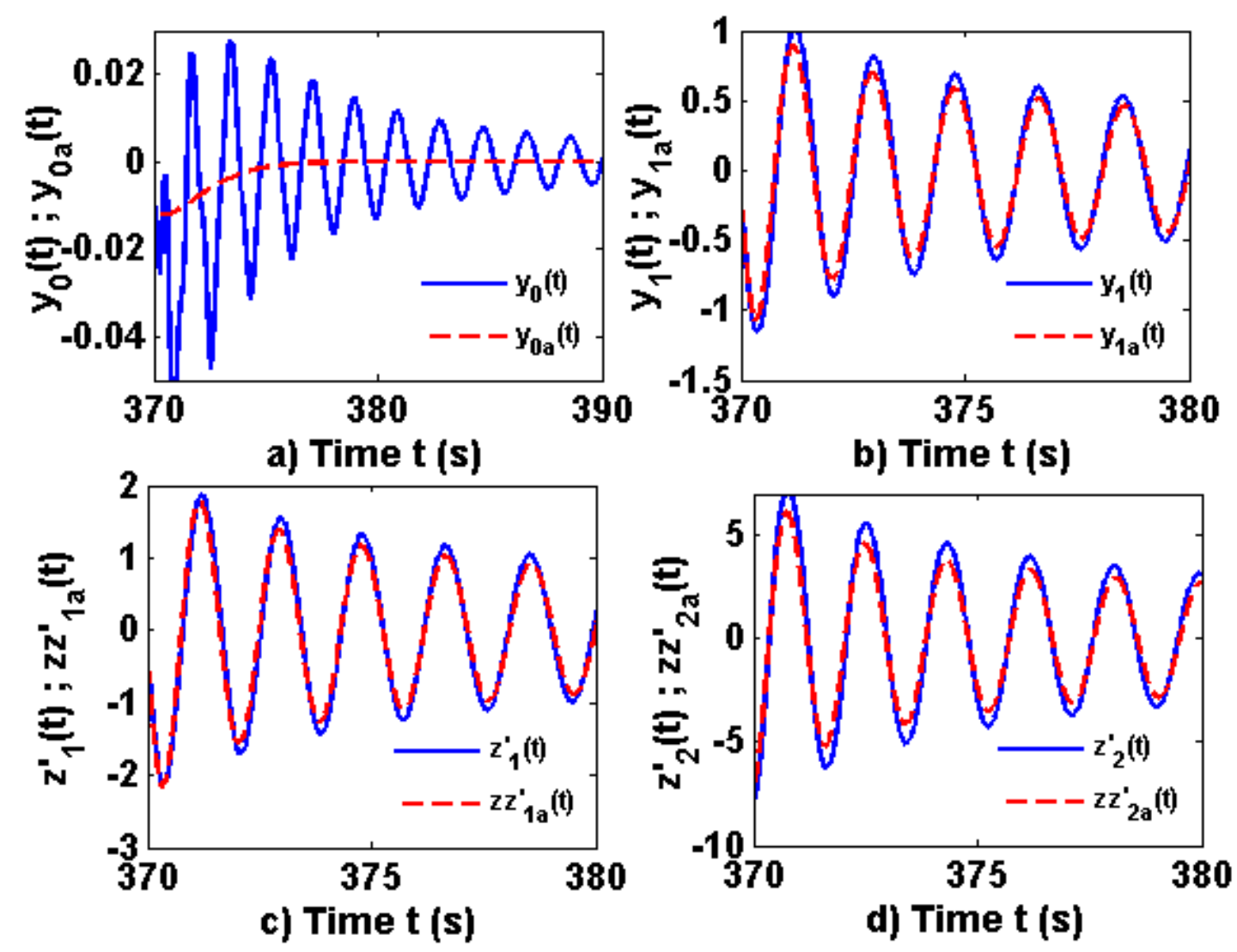

Figure 15 


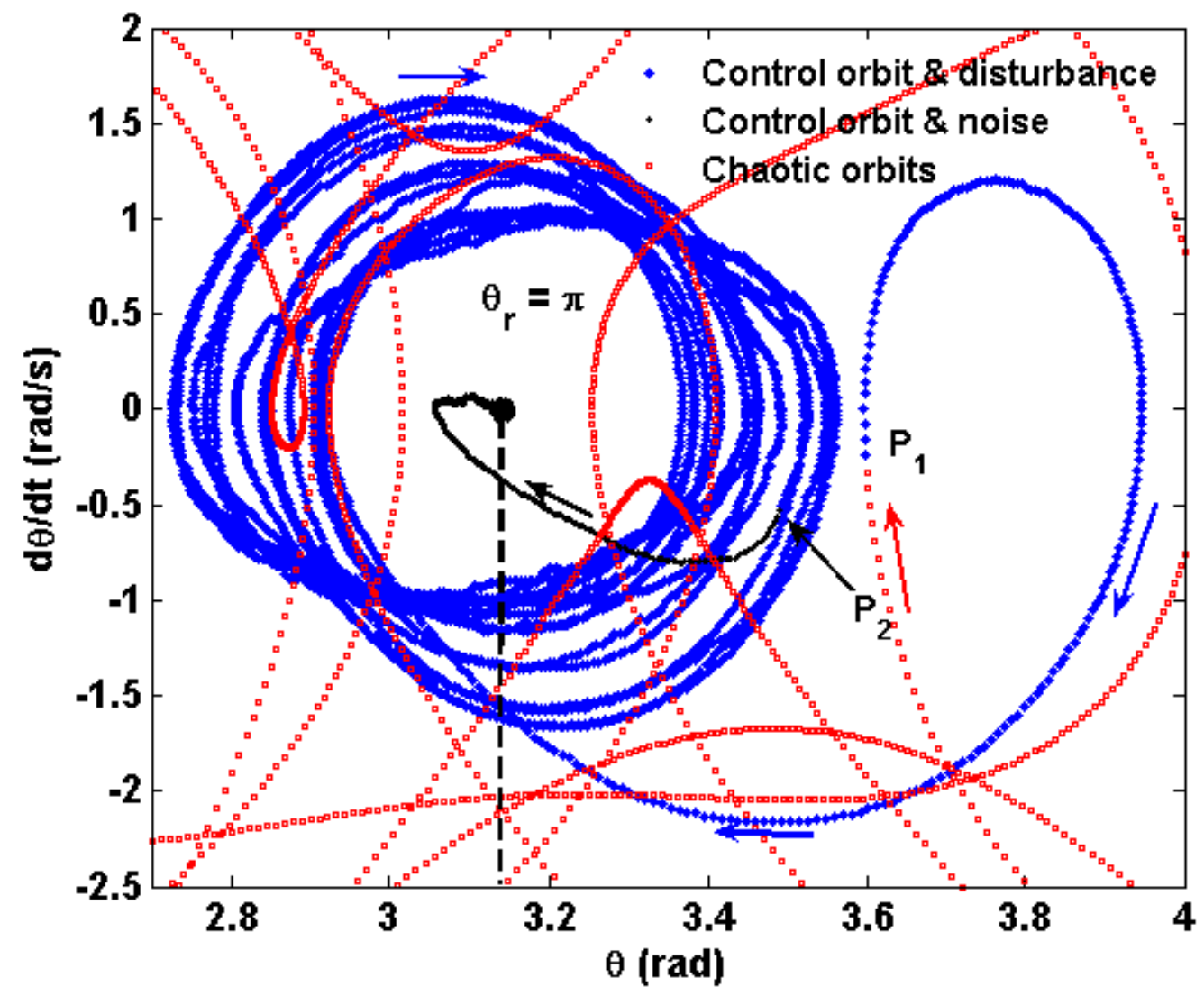

Figure 16 


\section{Figure captions}

Figure 1. Scheme of the pendulum formed by a rod of negligible mass connected to a hanging bob. The suspension point is subjected to vertical and horizontal harmonic disturbances. The parameter values of the system are $\mathrm{m}=0.5 \mathrm{~kg}, 1=1 \mathrm{~m}$ and $\mathrm{b}=0.5$ or $5 \mathrm{~N} \cdot \mathrm{m} \cdot \mathrm{s} / \mathrm{rad}$.

Figure 2. Simulation results without control torque and with vertical harmonic disturbances. The parameter values are $\omega_{0}^{2}=9.8 \mathrm{~m}^{2} / \mathrm{s}^{4}, \mathrm{~A}_{\mathrm{y}}=0.5 \mathrm{~m}, \omega_{\mathrm{yL}}=8.8544 \mathrm{rad} / \mathrm{s}$ and $\omega_{\mathrm{y}}=10>8.8544 \mathrm{rad} / \mathrm{s}$. The fourth-order Runge-Kutta integration method with a simulation step $\mathrm{T}=0.002 \mathrm{~s}$ has been used. a) State variables $\theta(\mathrm{t}), \mathrm{d} \theta(\mathrm{t}) / \mathrm{dt}$ and $\mathrm{u}(\mathrm{t})$ as a function of the time assuming initial conditions $\theta(0)=2.9 \mathrm{rad}$ and $\mathrm{d} \theta(0) / \mathrm{dt}=0 . \mathrm{b}$ ) Sensitive dependence for two simulations of $\theta(t)$ with initial conditions differing in $10^{-7}$ rad. c) Required forces $\mathrm{F}_{0 \mathrm{x}}$ and $\mathrm{F}_{0 \mathrm{y}}$ to produce the movements which appear in graphics a) and $b)$. d) State variables $\theta(t), d \theta(t) / d t$ and $u(t)$ as a function of the time assuming the presence of noise with an amplification factor $\mathrm{f}_{\mathrm{na}}=0.4(\mathrm{Eq}(15))$.

Figure 3. Simulation results with control torque and vertical harmonic disturbances. The parameter values of the system are $\omega_{0}^{2}=9.8 \mathrm{~m}^{2} / \mathrm{s}^{4}, \mathrm{~A}_{\mathrm{y}}=0.5 \mathrm{~m}, \omega_{\mathrm{yL}}=8.8544 \mathrm{rad} / \mathrm{s}$ and $\omega_{\mathrm{y}}=10>8.8544 \mathrm{rad} / \mathrm{s}$. The fourth-order Runge-Kutta integration method with a simulation step $\mathrm{T}=0.002 \mathrm{~s}$ has been used. a) State variables $\theta(\mathrm{t}), \mathrm{d} \theta(\mathrm{t}) / \mathrm{dt}$ and $\mathrm{u}(\mathrm{t})$ as a function of the time. b) Pendulum position in the presence of noise. c) Required forces $\mathrm{F}_{0 \mathrm{x}}$ and $\mathrm{F}_{0 \mathrm{y}}$ to produce the movements that appear in graphics a) and $\mathrm{b}$ ).

Figure 4. Simulation results without control torque and with horizontal harmonic disturbances of high frequency. The parameter values of the system are $\omega_{0}^{2}=9.8 \mathrm{~m}^{2} / \mathrm{s}^{4}$, $\theta_{\mathrm{d}}=0.8 \mathrm{rad}$ (desired tilt angle for the pendulum), $\mathrm{A}_{\mathrm{x}} \omega_{\mathrm{x}}=5.3040 \mathrm{~m} / \mathrm{s}$ (value to achieve stability), $A_{x}=0.035 \mathrm{~m}, \omega_{x}=151.5427 \mathrm{rad} / \mathrm{s}$ (frequency for stability) and $t_{d}=3 \mathrm{~s}$. The fourth-order Runge-Kutta integration method with a simulation step $\mathrm{T}=0.001 \mathrm{~s}$ has been used. a) State variable $\theta(t)$ as a function of the time assuming initial conditions $\theta(0)=0.5$ and $\mathrm{d} \theta(0) / \mathrm{dt}=0 . \mathrm{b})$ Required forces $\mathrm{F}_{0 \mathrm{x}}$ and $\mathrm{F}_{0 \mathrm{y}}$ to produce the movement that appears in graphic a).

Figure 5. a) Values of $b^{0}, b^{1}=b_{r}^{1}+i \cdot b_{i}^{1}\left(b_{r}^{1}=\operatorname{Re}\left[b^{1}\right], b_{i}^{1}=\operatorname{Im}\left[b^{1}\right]\right)$ and $\left|b^{1}\right|$ as $a$ function of the damping coefficient $\delta$. b) Values of the reset time $\tau_{\mathrm{i}}$ and the derivative constant $\tau_{\mathrm{d}}$ as a function of the damping coefficient $\delta$.

Figure 6. a) Real values of $x_{1}(0)$ and $y_{1}(0)$ deduced from the normalizing transformation and the inverse normalizing transformation as a function of the damping coefficient $\delta . b)$ Imaginary values of $\mathrm{x}_{1}(0)$ and $\mathrm{y}_{1}(0)$ deduced from the normalizing transformation and the inverse normalizing transformation as a function of the damping coefficient $\delta$. c) Values of $\mathrm{x}_{0}(0)$ and $\mathrm{y}_{0}(0)$ deduced from the normalizing transformation and the inverse normalizing transformation as a function of the damping coefficient $\delta$. d) Phase $F_{y 1}(t)$ obtained from the analytical expression of $y_{1 a}(t)$ as a function of the damping coefficient $\delta$. 
Figure 7. Simulation results with control torque and without harmonic disturbances. The parameter values of the system are $\omega_{0}^{2}=9.8 \mathrm{~m}^{2} / \mathrm{s}^{4}, \mathrm{~A}_{\mathrm{y}}=0.5 \mathrm{~m}, \mathrm{f}=10, \mathrm{~K}_{\mathrm{p}}=5.4444$ N.m, $\tau_{\mathrm{i}}=0.9989 \mathrm{~s}, \tau_{\mathrm{d}}=10^{-3} \mathrm{~s}, \mathrm{~b}^{0}=0.1614$ and $\mathrm{b}^{1}=-0.084+0.0084 \mathrm{i}$. The fourth-order Runge-Kutta integration scheme with a simulation step of $\mathrm{T}=0.005 \mathrm{~s}$ has been used. a) State variables $\theta(\mathrm{t}), \mathrm{d} \theta(\mathrm{t}) / \mathrm{dt}$ and $\mathrm{u}(\mathrm{t})$ as a function of the time assuming the initial conditions $\theta(0)=1.4 \mathrm{rad}$ and $\mathrm{d} \theta(0) / \mathrm{dt}=0$. b) Oscillation of $\theta(\mathrm{t})$ around the pointing-up position. c) Stable weak focus in the phase plane $\theta(\mathrm{t})-\mathrm{d} \theta(\mathrm{t}) / \mathrm{dt}$.

Figure 8. a) Values of $y_{1}(t)$ deduced from the inverse normalizing transformation and numerical results obtained from the simulation of the pendulum equations compared to $\mathrm{y}_{1 \mathrm{a}}(\mathrm{t})$. b) Values of $\mathrm{y}_{0}(\mathrm{t})$ deduced from the inverse normalizing transformation and numerical results obtained from the simulation of the pendulum equations compared to $\mathrm{y}_{0 \mathrm{a}}(\mathrm{t})$. The parameter values are indicated in the legend of Fig. 7.

Figure 9. a) Approximate and exact amplitudes for the radius of the weak focus depicted in Fig $7 \mathrm{c}$ ), which are deduced from the inverse normalizing transformation $y_{1}(t)$ and the analytical values of $y_{1 a}(t)$ respectively. Time evolution of the radius of the weak focus plotted in $\mathrm{f}$ Fig $7 \mathrm{c}$ ). b) Approximate and exact amplitudes of the deviation variable $z^{\prime}{ }_{1}(t)=\theta(t)-\pi$ as a function of the time. c) Approximate and exact amplitudes of the deviation variable $z_{2}{ }_{2}(t)=d \theta(t) / d t$ as a function of the time. The parameter values are indicated in the legend of Fig. 7.

Figure 10. a) Approximate phases $\mathrm{F}_{\mathrm{y} 1}(\mathrm{t})$ and exact phases $\mathrm{F}_{\mathrm{y} 1 \mathrm{a}}(\mathrm{t})$ deduced from the approximations $x_{1}(t) \approx y_{1}(t)$ and $x_{0}(t) \approx y_{0}(t)$ as well as from the analytical expression for $\mathrm{y}_{1 \mathrm{a}}(\mathrm{t})$. $\left.\mathrm{b}\right)$ Deviation variable $\mathrm{z}_{1}{ }_{1}(\mathrm{t})=\theta(\mathrm{t})-\pi$ and variable $\mathrm{zz}_{1}{ }_{1}(\mathrm{t})$ deduced from the variables $\mathrm{x}_{1}(\mathrm{t}), \mathrm{x}_{-1}(\mathrm{t})$ and $\mathrm{x}_{0}(\mathrm{t})$, which have been obtained from the normalizing transformation as a function of the time. c) Deviation variable $\mathrm{z}_{2}{ }_{2}(\mathrm{t})=\mathrm{d} \theta(\mathrm{t}) / \mathrm{dt}$ and variable $\mathrm{zz}_{2}{ }_{2}(\mathrm{t})$ deduced from the variables $\mathrm{x}_{1}(\mathrm{t}), \mathrm{x}_{-1}(\mathrm{t})$ and $\mathrm{x}_{0}(\mathrm{t})$, which have been obtained from the normalizing transformation as a function of the time. d) Deviation variable $z_{3}{ }_{3}(t)=u(t)$ and variable $z z_{3}(t)$ deduced from the variables $x_{1}(t), x_{-1}(t)$ and $\mathrm{x}_{0}(\mathrm{t})$, which have been obtained from the normalizing transformation as a function of the time. The parameter values are indicated in the legend of Fig. 7.

Figure 11. a) Values of the amplitude $A_{x}$ as a function of the frequency $\omega_{x}$ for different values of $A_{y}$ when the Melnikov function has no zeros. b) Values of the amplitude $A_{y}$ as a function of the frequency $\omega_{y}$ for different values of $A_{x}$ when the Melnikov function has no zeros. The values of points $\mathrm{P}_{1}$ and $\mathrm{P}_{2}$ could provide chaotic behavior, since the Melnikov function has zeros. However, the values of points $\mathrm{P}_{3}$ and $\mathrm{P}_{4}$ lead to a nonchaotic behavior since the Melnikov function has no zeros.

Figure 12. Strange attractor in the phase plane $\theta(t)-d \theta(t) / d t$. For $t<20 \mathrm{~s}$ the pendulum exhibits free oscillations. For $\mathrm{t}>20 \mathrm{~s}$ the harmonic disturbances are activated assuming the values $A_{x}=0.9 \mathrm{~m}, \omega_{x}=3.77 \mathrm{rad} / \mathrm{s}, A_{y}=0.8 \mathrm{~m}$ and $\omega_{y}=4.89 \mathrm{rad} / \mathrm{s}$ (points $P_{1}$ and $P_{2}$ of Fig 11). At $t=320 \mathrm{~s}$ the seek time is initialized, and afterwards a chaotic orbit intersects the capture region $\Omega\left(\mathrm{r}_{\mathrm{ax}}=0.5 \mathrm{rad}, \mathrm{r}_{\mathrm{ay}}=0.5 \mathrm{rad} / \mathrm{s}\right)$ at $\mathrm{t}=324.32 \mathrm{~s}$. The harmonic disturbances are removed at $\mathrm{t}=350 \mathrm{~s}$. The parameter values of the system are $\mathrm{f}=2, \mathrm{~K}_{\mathrm{p}}=9.8 \mathrm{~N} \cdot \mathrm{m}, \tau_{\mathrm{i}}=1.9615 \mathrm{~s}$ and $\tau_{\mathrm{d}}=10^{-3} \mathrm{~s}$. The fourth-order Runge-Kutta integration method with simulation step $\mathrm{T}=0.005 \mathrm{~s}$ has been employed. 
Figure 13. Simulation results obtained through the fourth-order Runge-Kutta method taking a simulation time of $450 \mathrm{~s}$ and a simulation step of $0.005 \mathrm{~s}$. a) Sensitive dependence for $\theta(\mathrm{t})$, which has been obtained from two simulations with initial conditions differing in $10^{-8}$. b) Lyapunov exponents as a function of the time, which provide an indicator of chaos because of the positive sign of one of them. c) Power spectral density of $\theta(\mathrm{t})$, which shows a wide band spectrum with the characteristic energy decay of chaotic systems. d) Required forces $F_{0 x}$ and $F_{0 y}$ to produce the movement depicted in graphic a). The parameter values are indicated in the legends of Fig 12.

Figure 14. Simulation results obtained through the fourth-order Runge-Kutta method taking a simulation time of $50000 \mathrm{~s}$ and a simulation step of $0.009 \mathrm{~s}$. The parameter values of the system are $A_{x}=0.9 \mathrm{~m}, \omega_{x}=3.77 \mathrm{rad} / \mathrm{s}, A_{y}=0.8 \mathrm{~m}$ and $\omega_{y}=4.89 \mathrm{rad} / \mathrm{s}$ (points $\mathrm{P}_{1}$ and $\mathrm{P}_{2}$ of Fig 11). a) Angular variable $\mathrm{x}_{5} \equiv \mathrm{f}_{\omega \mathrm{y}}\left(0<\mathrm{f}_{\omega \mathrm{y}}<2 \pi\right)$ associated to the harmonic vertical disturbance (with frequency $\omega_{\mathrm{y}}$ ) as a function of $\theta(\mathrm{t})$. b) Angular variable $\mathrm{x}_{5} \equiv \mathrm{f}_{\omega \mathrm{y}}$ as a function of $\mathrm{d} \theta(\mathrm{t}) / \mathrm{dt}$. c) Poincaré section for $\mathrm{f} \omega_{\mathrm{y} 1}=4$. d) Poincaré section for $\mathrm{f} \omega_{\mathrm{y} 2}=2$.

Figure 15. Values of $y_{1}(t)$ deduced from the inverse normalizing transformation and numerical results deduced from the simulation of the pendulum equations compared against the analytical expression of $\left.\mathrm{y}_{1 \mathrm{a}}(\mathrm{t}) \mathrm{b}\right)$ Values of $\mathrm{y}_{0}(\mathrm{t})$ deduced from the inverse normalizing transformation and numerical results deduced from the simulation of the pendulum equations compared against the analytical expression of $\mathrm{y}_{0 \mathrm{a}}(\mathrm{t})$. c) Deviation variable $z^{\prime}{ }_{1}(\mathrm{t})=\theta(\mathrm{t})-\pi$ and variable $\mathrm{zz}^{\prime}{ }_{1}(\mathrm{t})$ deduced from the variables $\mathrm{x}_{1}(\mathrm{t}), \mathrm{x}_{-1}(\mathrm{t})$ and $\mathrm{x}_{0}(\mathrm{t})$, which have been obtained from the normalizing transformation as a function of the time. d) Deviation variable $z^{\prime}{ }_{2}(t)=d \theta(t) / d t$ and variable $z z_{2}{ }_{2}(t)$ deduced from the variables $\mathrm{x}_{1}(\mathrm{t}), \mathrm{x}_{-1}(\mathrm{t})$ and $\mathrm{x}_{0}(\mathrm{t})$, which have been obtained from the normalizing transformation as a function of the time. The parameter values are indicated in the legend of Fig 12.

Figure 16. Strange attractor in the phase plane $\theta(\mathrm{t})-\mathrm{d} \theta(\mathrm{t}) / \mathrm{dt}$. Simulation results obtained through the fourth-order Runge-Kutta method taking a simulation time of $450 \mathrm{~s}$ and a simulation step of $0.005 \mathrm{~s}$ for $\mathrm{A}_{\mathrm{x}}=0.9 \mathrm{~m}, \omega_{\mathrm{x}}=3.77 \mathrm{rad} / \mathrm{s}, \mathrm{A}_{\mathrm{y}}=0.8 \mathrm{~m}$ and $\omega_{\mathrm{y}}=4.89$ $\mathrm{rad} / \mathrm{s}$ (points $\mathrm{P}_{1}$ and $\mathrm{P}_{2}$ of Fig 11). At $\mathrm{t}=320 \mathrm{~s}$ the seek time is initialized, and afterwards a chaotic orbit intersects the capture region $\Omega\left(\mathrm{r}_{\mathrm{ax}}=0.5 \mathrm{rad}, \mathrm{r}_{\mathrm{ay}}=0.5 \mathrm{rad} / \mathrm{s}\right)$ at $\mathrm{t}=322.69 \mathrm{~s}$, instant at which the PID parameter values are set to $\mathrm{f}=2, \mathrm{~K}_{\mathrm{p}}=9.8 \mathrm{~N} \cdot \mathrm{m}, \tau_{\mathrm{i}}$ $=1.9615 \mathrm{~s}$ and $\tau_{\mathrm{d}}=10^{-3} \mathrm{~s}$. For $\mathrm{t} \geq 350 \mathrm{~s}$ the PID parameters are changed to $\mathrm{K}_{\mathrm{p}}=18.1183$ N.m, $\tau_{\mathrm{i}}=1.6850 \mathrm{~s}, \tau_{\mathrm{d}}=0.2668 \mathrm{~s}$ and noise with an amplification factor $\mathrm{f}_{\mathrm{na}}=0.5$ is added. 